\title{
Plasma proteome and metabolome characterization of an experimental human thyrotoxicosis model
}

\author{
Maik Pietzner ${ }^{1,2 \dagger}$, Beatrice Engelmann ${ }^{3}$, Tim Kacprowski ${ }^{2,3}$, Janine Golchert ${ }^{3}$, Anna-Luise Dirk ${ }^{4}$, Elke Hammer ${ }^{2,3}$, \\ K. Alexander Iwen ${ }^{4}$, Matthias Nauck ${ }^{1,2}$, Henri Wallaschofski ${ }^{1,5}$, Dagmar Führer ${ }^{6}$, Thomas F. Münte ${ }^{7}$, \\ Nele Friedrich ${ }^{1,2,8}$, Uwe Völker ${ }^{2,3,9}$, Georg Homuth ${ }^{3,9^{*+}}$ and Georg Brabant ${ }^{4^{*}+}$
}

\begin{abstract}
Background: Determinations of thyrotropin (TSH) and free thyroxine $\left(\mathrm{FT}_{4}\right)$ represent the gold standard in evaluation of thyroid function. To screen for novel peripheral biomarkers of thyroid function and to characterize $\mathrm{FT}_{4}$-associated physiological signatures in human plasma we used an untargeted OMICS approach in a thyrotoxicosis model.

Methods: A sample of 16 healthy young men were treated with levothyroxine for 8 weeks and plasma was sampled before the intake was started as well as at two points during treatment and after its completion, respectively. Mass spectrometry-derived metabolite and protein levels were related to $\mathrm{FT}_{4}$ serum concentrations using mixed-effect linear regression models in a robust setting. To compile a molecular signature discriminating between thyrotoxicosis and euthyroidism, a random forest was trained and validated in a two-stage cross-validation procedure.

Results: Despite the absence of obvious clinical symptoms, mass spectrometry analyses detected 65 metabolites and 63 proteins exhibiting significant associations with serum $\mathrm{FT}_{4}$. A subset of 15 molecules allowed a robust and good prediction of thyroid hormone function ( $\mathrm{AUC}=0.86$ ) without prior information on $\mathrm{TSH}_{\mathrm{H}}$ or FT 4 . Main $\mathrm{FT}_{4}$-associated signatures indicated increased resting energy expenditure, augmented defense against systemic oxidative stress, decreased lipoprotein particle levels, and increased levels of complement system proteins and coagulation factors. Further association findings question the reliability of kidney function assessment under hyperthyroid conditions and suggest a link between hyperthyroidism and cardiovascular diseases via increased dimethylarginine levels.
\end{abstract}

Conclusion: Our results emphasize the power of untargeted OMICS approaches to detect novel pathways of thyroid hormone action. Furthermore, beyond $\mathrm{TSH}_{\mathrm{H}}$ and $\mathrm{FT}_{4}$, we demonstrated the potential of such analyses to identify new molecular signatures for diagnosis and treatment of thyroid disorders. This study was registered at the German Clinical Trials Register (DRKS) [DRKS00011275] on the 16th of November 2016.

Keywords: Hyperthyroidism, Metabolomics, Proteomics, Thyroid function, Thyrotoxicosis

\footnotetext{
*Correspondence: georg.homuth@uni-greifswald.de; georg.brabant@uksh.de

${ }^{\dagger}$ Equal contributors

${ }^{3}$ Department of Functional Genomics, Interfaculty Institute for Genetics and Functional Genomics, University Medicine and Ernst-Moritz-Arndt University Greifswald, Friedrich-Ludwig-Jahn-Straße 15a, D-17475 Greifswald, Germany ${ }^{4}$ Medical Clinic I, University of Lübeck, Experimental and Clinical

Endocrinology, Ratzeburger Allee 160, Zentralklinikum (Haus 40), 23538 Lübeck, Germany

Full list of author information is available at the end of the article
} 


\section{Background}

Thyroid hormones $(\mathrm{TH})$ circulating as thyroxine $\left(\mathrm{T}_{4}\right)$ and triiodothyronine $\left(\mathrm{T}_{3}\right)$ are essential for normal development and function of virtually all tissues [1]. Both their synthesis and release are closely controlled by pituitary thyroid stimulating hormone (TSH), which in turn is stimulated by hypothalamic thyrotropin releasing hormone (TRH). TH exert a negative feedback on synthesis and secretion of TRH as well as of TSH. As the feedback of TH on the hypothalamic-pituitary regulation of TSH is particularly sensitive, the robust relation of TSH and free $\mathrm{T}_{4}\left(\mathrm{FT}_{4}\right)$ is generally used as the 'gold standard' tool for diagnosis and follow-up of thyroid disorders.

Specific TH transporters mediate the cellular uptake of $\mathrm{TH}$ [2]. At the latest in the target cells, specific deiodinases convert $T_{4}$ to $T_{3}$ which is the major ligand for the nuclear TH receptors (TR) $\alpha$ and $\beta$ and their subtypes [1]. Formation of ligand-activated TR homodimers and heterodimers with TR auxiliary proteins and other receptors, such as retinoid $\mathrm{X}$ receptor (RXR), finally results in stimulated or repressed expression of $\mathrm{TH}$ target genes. In addition to this so-called genomic action mediated by nuclear TRs, TH exert rapid non-genomic effects by binding to extranuclear receptors, like truncated cytoplasmic TR $\alpha$ isoforms or plasma membrane-localized integrin $\alpha v \beta 3$, resulting in the activation of specific phosphorylation cascades [3]. Also, for the putative $\mathrm{TH}$ derivative 3,5-diiodothyronine, interaction with specific mitochondrial sites was reported [3]. Thus, in sum, by cell- and organ-specific $\mathrm{TH}$ uptake and activation, TR subtype synthesis and nongenomic modulation, $\mathrm{TH}$ are able to induce their various tissue- and cell-specific responses. It is thus not surprising that clinical symptoms of thyroid dysfunction are regarded to be of restricted diagnostic value because they are neither sufficiently sensitive nor specific [4]. Currently, the diagnosis of thyroid dysfunction and the assessment of treatment effects are almost entirely based on the biochemical determination of serum $\mathrm{TSH}$, free $\mathrm{T}_{4}\left(\mathrm{FT}_{4}\right)$ and, under special conditions, free $\mathrm{T}_{3}\left(\mathrm{FT}_{3}\right)$. However, their use is limited by a number of drawbacks.

Despite the sensitive negative feedback regulation between TSH and $\mathrm{FT}_{4}$ leading to a tightly controlled individual set point $[5,6]$, large population-based studies established a wide reference range for TSH and free TH levels. This is explained by varying sensitivity at different levels of the activation process as well as the negative feedback mechanisms [7] and differences between assay specificities $[8,9]$. Additionally, a number of rare severe clinical conditions lead to discordant alterations in serum $\mathrm{TSH}$ and $\mathrm{FT}_{4}$, including resistance to $\mathrm{TH}$, TSH producing pituitary tumors, or central hypothyroidism $[10,11]$. Therefore, peripheral biomarkers such as cholesterol and sex hormone- binding globulin (SHBG) concentrations have been suggested under these conditions as they are strongly correlated with thyroid function [12, 13]. However, because these parameters are also influenced by non-thyroidal disturbances, they were never established in clinical practice and accordingly current guidelines do not recommend their use [14]. Thus, currently available diagnostic tools are insufficient and novel biomarkers are urgently needed.

Indeed, systematic screens for novel markers of thyroid function in humans are lacking so far. In particular, only few studies attempted to detect peripheral $\mathrm{TH}$ effects by untargeted approaches. The influence of thyroid dysfunction on various tissue-specific proteomes or the metabolomes of serum and urine was assessed almost entirely using rodent models [15-18]. Even if these studies undoubtedly added to our understanding of $\mathrm{TH}$ action on metabolism, translation of these results to humans is still missing. Moreover, most of the scarce data on peripheral $\mathrm{TH}$ effects in humans are based on observations in patients with thyroid disorders such as autoimmune thyroid disease, which hamper the distinction between $\mathrm{TH}$ dependent effects and those related to the underlying autoimmune process. To avoid these problems, we herein studied $\mathrm{TH}$ effects in a strictly controlled model of experimental hyperthyroidism where healthy young male volunteers were subjected to a challenge of thyroxine over a period of 8 weeks. Untargeted plasma proteome and metabolome analyses were performed in a hypothesis-free approach to detect $\mathrm{FT}_{4}$-associated proteins and metabolites, and the generated data were used for characterization of main physiological signatures and to develop a biomarker-based classification model that allows prediction of $\mathrm{TH}$ function without prior information on TSH or free $\mathrm{TH}$.

\section{Methods}

\section{Study design and sampling}

Sixteen young healthy male subjects were treated with a single tablet of $250 \mu \mathrm{g}$ levothyroxine ( $\mathrm{L}-\mathrm{T}_{4}$; Henning-Berlin, Berlin, Germany) per day for 8 weeks. Plasma was sampled before $\mathrm{L}-\mathrm{T}_{4}$ intake started (baseline, bas), after 4 (w4(T4)) and 8 (w8(T4)) weeks under treatment as well as 4 (w12) and 8 (w16) weeks after ending the application, respectively (Fig. 1a). The chosen sample size is appropriate as the volunteers were selected to reduce inter-individual variance. The repeated measure character of the study further reduced the influence of inter-individual variance. Body mass index (BMI) of the volunteers ranged from 21 to $30 \mathrm{~kg} / \mathrm{m}^{2}$ and their age from 22 to 34 years (Table 1). During the study, thyrotoxicosis questionnaires were performed as well as $24 \mathrm{~h}$ blood pressure, and pulse rate activity (Cambridge Nanotechnology, Cambridge, UK) were recorded. The work has been approved by the ethics committee of the University of Lübeck and written informed consent was received from all participants prior to the study. The study conformed to the WMA Declaration of Helsinki. 

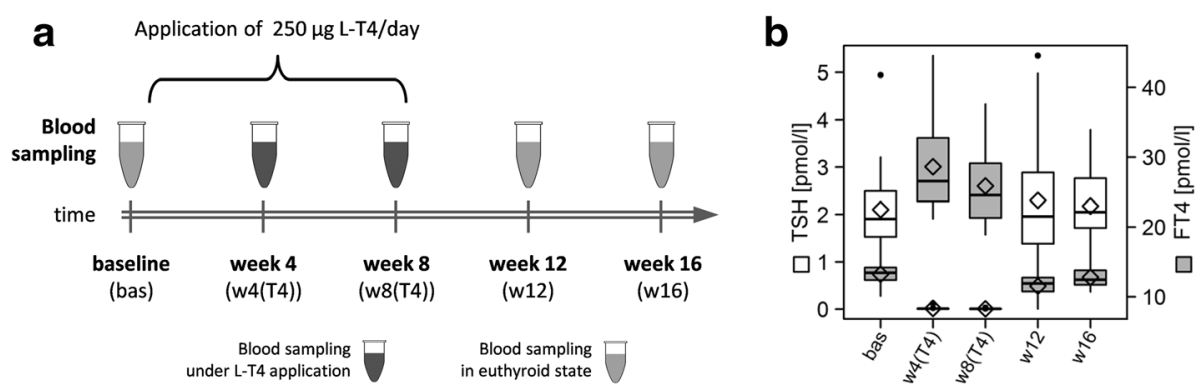

Fig. 1 a Study design including sampling time points as well as duration of levothyroxine $\left(L-T_{4}\right)$ treatment. b Boxplots with mean values (diamonds) of serum TSH (white) and $\mathrm{FT}_{4}$ (grey) for each time point. bas baseline, w4(T4)/w8(T4) 4 and 8 weeks of levothyroxine treatment, w12/ w16 4 and 8 weeks after stopping the application point

\section{Assays}

Serum levels of TSH, free triiodothyronine $\left(\mathrm{FT}_{3}\right)$ and $\mathrm{FT}_{4}$ were measured using an immunoassay (Dimension VISTA, Siemens Healthcare Diagnostics, Eschborn, Germany) with a functional sensitivity of $0.005 \mathrm{mU} / \mathrm{L}$ for $\mathrm{TSH}, 0.77 \mathrm{pmol} / \mathrm{L}$ for $\mathrm{FT}_{3}$, and $1.3 \mathrm{pmol} / \mathrm{L}$ for $\mathrm{FT}_{4}$. SHBG levels were determined via a chemiluminescent enzyme immunoassay on an Immulite 2000XPi analyzer (SHBG Immulite 2000, Siemens Healthcare Medical Diagnostics, Bad Nauheim, Germany) with a functional sensitivity of $0.02 \mathrm{nmol} / \mathrm{L}$. Serum cystatin C (CYTC) was measured using a nephelometric assay (Dimension VISTA, Siemens Healthcare Diagnostics, Eschborn, Germany) with a functional sensitivity of $0.05 \mathrm{mg} / \mathrm{L}$. Insulin serum concentrations were measured using a chemiluminescent immunometric assay (Immulite $200 \mathrm{XPi}$; Siemens Healthcare Diagnostics) with a functional sensitivity of $2 \mathrm{mU} / \mathrm{L}$. Lipids (total cholesterol, HDL- and LDL cholesterol, triglycerides), serum glucose, serum activities of alanine amino transferase (ALT), aspartate amino transferase (AST), $\gamma$-glutamyl transpeptidase (GGT), as well as the levels of the complement factors $\mathrm{C} 3$ and $\mathrm{C} 4$ were measured by standard methods (Dimension VISTA, Siemens Healthcare Diagnostics, Eschborn, Germany).

\section{Plasma metabolome analysis}

Metabolic profiling of plasma samples was performed by Metabolon Inc. (Durham, NC, USA), a commercial supplier of metabolic analyses. Three separate analytical methods (GC-MS and LC-MS (positive and negative mode)) were used to detect a broad metabolite panel [19]. Briefly, proteins were precipitated from $100 \mu \mathrm{L}$ plasma with methanol, which further contained four standards to monitor extraction efficiency, using an automated liquid handler (Hamilton ML STAR, Hamilton Company, Salt Lake City, UT, USA). The resulting extract was divided into four aliquots; two for analysis by LC, one for analysis by GC, and one reserve aliquot. Aliquots were placed briefly on a TurboVap ${ }^{\oplus}$ (Zymark, Sparta, NJ, USA) to remove the organic solvent. Each aliquot was then frozen and dried under vacuum. LC-MS analysis was performed on a LTQ mass spectrometer (Thermo Fisher Scientific Inc., Waltham, MA, USA) equipped with a Waters Acquity UPLC system (Waters Corporation, Milford, MA, USA). Two aliquots were reconstituted either with $0.1 \%$ formic acid (positive mode) or $6.5 \mathrm{mM}$ ammonium bicarbonate (negative mode). Two separate columns $(2.1 \times 100 \mathrm{~mm}$ Waters BEH C18 $1.7 \mu \mathrm{m}$ particle $)$ were used for acidic (solvent A: $0.1 \%$ formic acid in $\mathrm{H}_{2} \mathrm{O}$, solvent B: $0.1 \%$ formic acid in methanol) and basic (A: 6.5 $\mathrm{nM}$ ammonium bicarbonate $\mathrm{pH}$ 8.0, B: $6.5 \mathrm{nM}$ ammonium bicarbonate in $98 \%$ methanol) mobile phase conditions, optimized for positive and negative electrospray ionization, respectively. After injection, the samples were separated in a gradient from $100 \%$ A to $98 \%$ B. The MS analysis alternated between MS and data-dependent MS/ MS scans using dynamic exclusion. GC-MS analysis was performed on a Finnigan Trace DSQ fast-scanning singlequadrupole mass spectrometer (Thermo Fisher Scientific Inc., Waltham, MA, USA), equipped with a GC column containing $5 \%$ phenyl residues. The temperature was ramped between 60 and $340{ }^{\circ} \mathrm{C}$. For electron impact ionization one aliquot was derivatized under dried nitrogen using bistrimethyl-silyl-triflouroacetemide. Quality control of platform performance was achieved by the use of pooled samples and technical blanks as well as the addition of non-interfering internal standards to the samples. Metabolites were identified from LC-MS and GC-MS spectra by automated comparison with a proprietary library, containing retention times, $\mathrm{m} / \mathrm{z}$ ratios, and related adduct/fragment spectra of over 1000 standard compounds measured by Metabolon. To correct for daily variations of platform performance, the raw area count of each metabolite was rescaled by the respective median value of the run day. In total, 380 metabolites could be identified.

\section{Plasma proteome analysis}

Depletion of six highly abundant proteins in plasma was performed using multi-affinity chromatography (MARS6- 


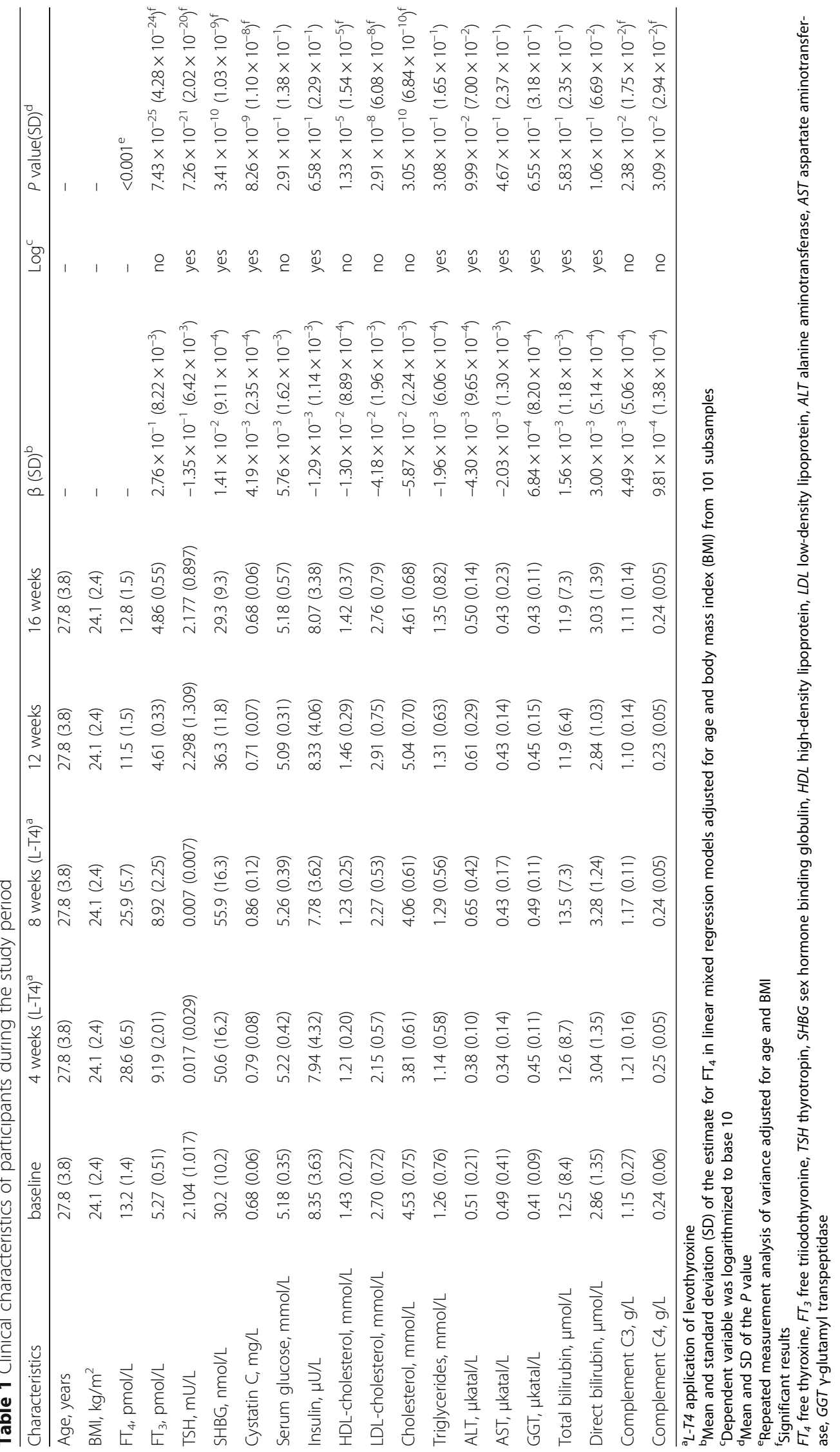


human, Agilent Technologies, Waldbronn, Germany) in accordance with the manufacturer's protocol. After precipitation of proteins of the non-bound fraction with trichloroacetic acid (final concentration 15\%), the pellet was re-suspended in $100 \mu \mathrm{L} 8 \mathrm{M}$ urea/2 M thiourea. Protein concentrations of depleted samples were determined via a Bradford Assay (Bio-Rad Laboratories, Munich, Germany) using bovine serum albumin as standard protein. Individual protein samples $(4 \mu \mathrm{g})$ were reduced with $2.5 \mathrm{mM}$ dithiothreitol $\left(60{ }^{\circ} \mathrm{C}, 1 \mathrm{~h}\right)$, subsequently alkylated with $10 \mathrm{mM}$ iodoacetamide $\left(37^{\circ} \mathrm{C}, 30 \mathrm{~min}\right)$, and subjected to proteolytic cleavage with trypsin (Promega, Madison, WI, USA) using a trypsin to protein ratio of $1: 25$ overnight at $37^{\circ} \mathrm{C}$. After stopping the digestion with $1 \%$ acetic acid, samples were purified with C18 ZipTip with a loading capacity of $2 \mu \mathrm{g}$ (Millipore Cooperation, Billerica, MA, USA). Prior to MS analysis, desalted peptides were subjected to reverse phase chromatography. Chromatographic separation of peptides was done on a nanoAquity UPLC system equipped with a pre-column (nano Aquity UPLC Trap column, $180 \mu \mathrm{m} \times$ $20 \mathrm{~mm}, 5 \mu \mathrm{m})$ and reverse phase column (BEH130 C18, $100 \mu \mathrm{m} \times 100 \mathrm{~mm}, 1.7 \mu \mathrm{m}$ ) configuration (Waters Corporation, Milford, MA, USA). A 100-min non-linear gradient of $2-60 \% \mathrm{ACN}$ in $0.1 \%$ acetic acid was run at a constant flow rate of $0.4 \mu \mathrm{L} / \mathrm{min}$. Mass spectral data were recorded on-line on a LTQ-Orbitrap Velos mass spectrometer (Thermo Electron, Bremen, Germany) which was operated in a data-dependent acquisition mode. MS/MS fragmentation was performed by collision induced dissociation. The recorded LC-MS/MS raw data were processed using the Refiner MS software version 7.6.6 (GeneData, Basel, Switzerland) with an adapted workflow with the following steps: (1) chemical noise removal, (2) retention time alignment across all samples, and (3) feature extraction and isotope group clustering. Data was searched against a human Swissprot/Uniprot database (rel. 2012/08) limited to human entries with a precursor ion tolerance set to $10 \mathrm{ppm}$ (0.6 Da for fragment ions) using an in-house MASCOT server (rel. 2.3). The carbamidomethylation of cysteine was set as static modification, methionine oxidation was considered as dynamic modification. Peptides identified with rank $=1$ and an ion score $\geq 20$ and identified as unique in the data set were used for relative quantitation on the level of summed peptide intensities per protein. MS analyses of all 80 plasma samples revealed 2374 unique peptides representing 497 human proteins. The mass spectrometry proteomics data have been deposited to the ProteomeXchange Consortium via the PRIDE [20] partner repository with the dataset identifier PXD004815 and 10.6019/PXD004815.

\section{Statistical analysis}

To ensure a median availability of data points on three time points, only metabolites and proteins with less than
$40 \%$ missing values were used for the analysis, resulting in 349 metabolites and 437 proteins. Values of metabolite/ protein intensities were $\log _{10}$-transformed. To account for compliance and intestinal resorption during $\mathrm{L}-\mathrm{T}_{4}$ treatment we applied a mixed-effect linear regression model with serum $\mathrm{FT}_{4}$ concentrations as exposure and metabolite/protein concentrations as outcome. Since the study considered repeated measurements, serum $\mathrm{FT}_{4}$ was determined as a fixed effect whereas the study participant was the random effect in the model. All analyses were adjusted for baseline age and BMI as well as experimental batch in case of proteome analyses. To account for multiple testing, we adjusted the $P$ values of the regression analysis by controlling the false discovery rate (FDR) at 5\% [21]. Distributional assumptions were tested visually using QQplots and no obvious violations were observed. Robustness of the results was assessed by a leave-three-out procedure. For this purpose, we randomly chose three participants and excluded them from the analyses. This procedure was repeated 100 times. Since three participants strikingly differed in their response to $\mathrm{L}_{-} \mathrm{T}_{4}$ regarding their serum ALT and AST activities, an additional data set was created excluding them leading to finally 101 distinct subsets of the data. Subsequently, estimates and FDR values were averaged across the subsamples. Metabolites and proteins with an average FDR below 0.05 were defined significant. In consequence, the results presented in this work constitute the most robust $\mathrm{FT}_{4}$-associated alterations. The functional classification analysis for significantly altered proteins was performed using Ingenuity Pathway Analysis software (Ingenuity Systems, Redwood City, CA, USA). Significance of the enrichment of altered proteins among functional categories was assessed by Fisher's exact test. For every time point, ratios to the baseline values for each participant were calculated and plotted as mean $\log _{2}$-fold change.

\section{Sample classification}

For classification purposes, samples were divided in two groups. First, all samples before ${\mathrm{L}-\mathrm{T}_{4}}_{4}$ treatment as well as 8 weeks after cessation of treatment were defined as euthyroid. Second, all samples from the two treatment time points were defined as hyperthyroid. Both assignments were justified by suppressed serum TSH concentrations in concordance with elevated serum $\mathrm{FT}_{4}$ (Table 1 and Fig. 1). In total, 64 samples were used for classification analysis (time point w12, 4 weeks after stop of treatment, was left out because of the presence of an intermediate state). To ensure reproducibility of possible markers, only metabolites/proteins without missing values as well as unambiguous assignment were used, resulting in 201 metabolites and 207 proteins. Since no independent validation set was available and to avoid overfitting, we performed a twostage cross validation procedure to select a subset of metabolites/proteins capable of classifying the samples 
using a random forest [22] as classifier (Additional file 1: Figure S1). A first split was performed to divide samples in training and validation set (outer loop; repeated 30 times). The resulting training sets were once more partitioned into training and test set (inner loop; repeated 50 times). Based on the last split, a random forest was trained. Prediction performance was assessed using receiver operating characteristic (ROC) curves on the independent test set for the current loop. Variable importance was assessed by the Gini index [23] for each feature of the trained forest. Variable importance of each inner loop were averaged weighted by the area under the ROC-curves (AUC). The 15 most important variables from the inner loop were taken forward to build a new random forest. Analogous to the previous procedure the prediction performance was assessed yielding the final classification performance based on a reduced subset of the features. The random forest was implemented in $\mathrm{R}$ via the randomForest package ( $\mathrm{v}$ 4.610) [22]. Statistical analyses were performed using SAS version 9.4 (SAS statistical software, version 9.4, SAS Institute, Inc.; NC, USA) and R 3.0.1 (R Foundation for statistical computing, version 3.0.1, Vienna, Austria).

\section{Results and Discussion}

\section{$\mathrm{L}^{-} \mathrm{T}_{4}$ treatment and standard clinical hormone assays}

As previously described [24], treatment with $250 \mu \mathrm{g} /$ day $\mathrm{L}-\mathrm{T}_{4}$ for 8 weeks resulted in the expected suppression of mean TSH concentrations from $2.10 \mathrm{mU} / \mathrm{L}$ (standard deviation (SD): \pm 1.01 ) at baseline to $0.017 \mathrm{mU} / \mathrm{L}$ (SD: $\pm 0.029)$ at 4 weeks and $0.007 \mathrm{mU} / \mathrm{L}$ (SD: \pm 0.007 ) at 8 weeks, respectively. Mean concentrations of $\mathrm{FT}_{4}$ and $\mathrm{FT}_{3}$ exhibited the opposite profile with peak concentrations of $28.6 \mathrm{pmol} / \mathrm{L}(\mathrm{SD}: 5.7$ ) and $9.19 \mathrm{pmol} / \mathrm{L}$ (SD: \pm 2.01 ) after 4 weeks of $\mathrm{L}_{-} \mathrm{T}_{4}$ intake, respectively, consistent with a biochemical condition of overt hyperthyroidism (Fig. 1 and Table 1). All parameters normalized within the first 4 weeks after termination of $\mathrm{L}^{-} \mathrm{T}_{4}$ intake (Fig. 1b). We further assessed effects on some of the well-known TH targets such as SHBG, CYTC, and different blood lipids (Table 1). In general, $\mathrm{L}-\mathrm{T}_{4}$ treatment resulted in a transient decline of blood lipids (Fig. 2) apart from triglycerides, whereas serum glucose and insulin were not significantly altered. The complement factors $\mathrm{C} 3$ and $\mathrm{C} 4$ showed a moderate, but significant positive association with $\mathrm{FT}_{4}$ (Fig. 2).

\section{General $\mathrm{FT}_{4}$-associated alterations of the plasma metabolome}

Treatment of the euthyroid male volunteers with $\mathrm{L}_{-} \mathrm{T}_{4}$ markedly affected the plasma metabolome, significantly altering the levels of 65 out of 349 detected metabolites (19\%), of which 45 exhibited a positive and 20 a negative association with serum $\mathrm{FT}_{4}$, respectively. The associated metabolites represented diverse metabolite classes, where lipids and related compounds encompassed the largest portion of $\mathrm{FT}_{4}$-associated molecules (39 of 65
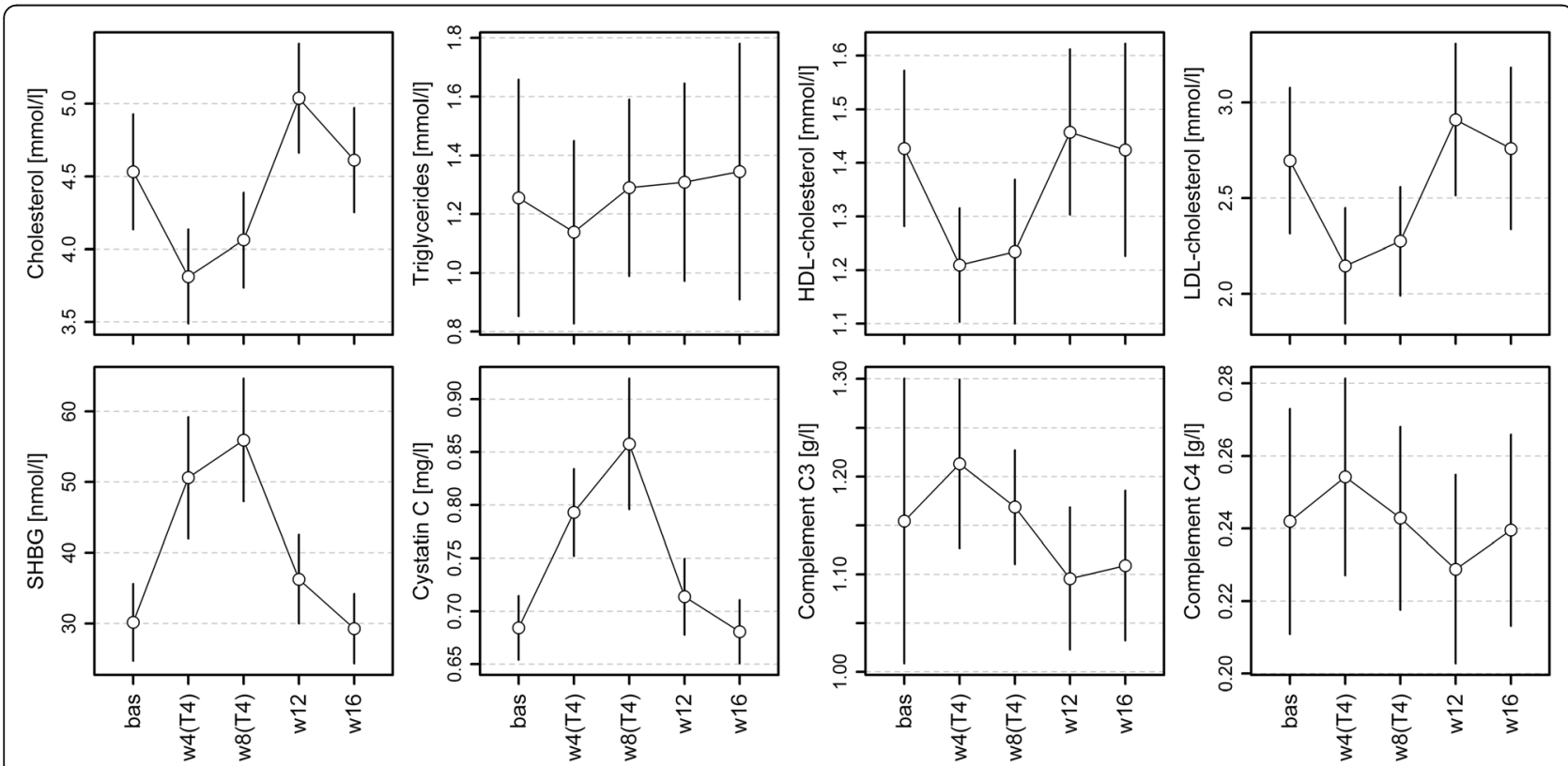

Fig. 2 Means with 95\% confidence intervals for serum concentrations of selected biochemical parameters during the study periods. Corresponding estimates from regression analyses are given in Table 1. bas baseline, w4(T4)/w8(T4) 4 and 8 weeks of levothyroxine treatment, w12/w16 4 and 8 weeks after stopping the application 
present in the analysis panel). These could be assigned to the following categories: free fatty acids (FFAs), acyl carnitines (ACs), polyunsaturated fatty acids (PUFAs), lysophospholipids (LPs), and androgens. All results are summarized in Fig. 3 and Additional file 2: Table S1.

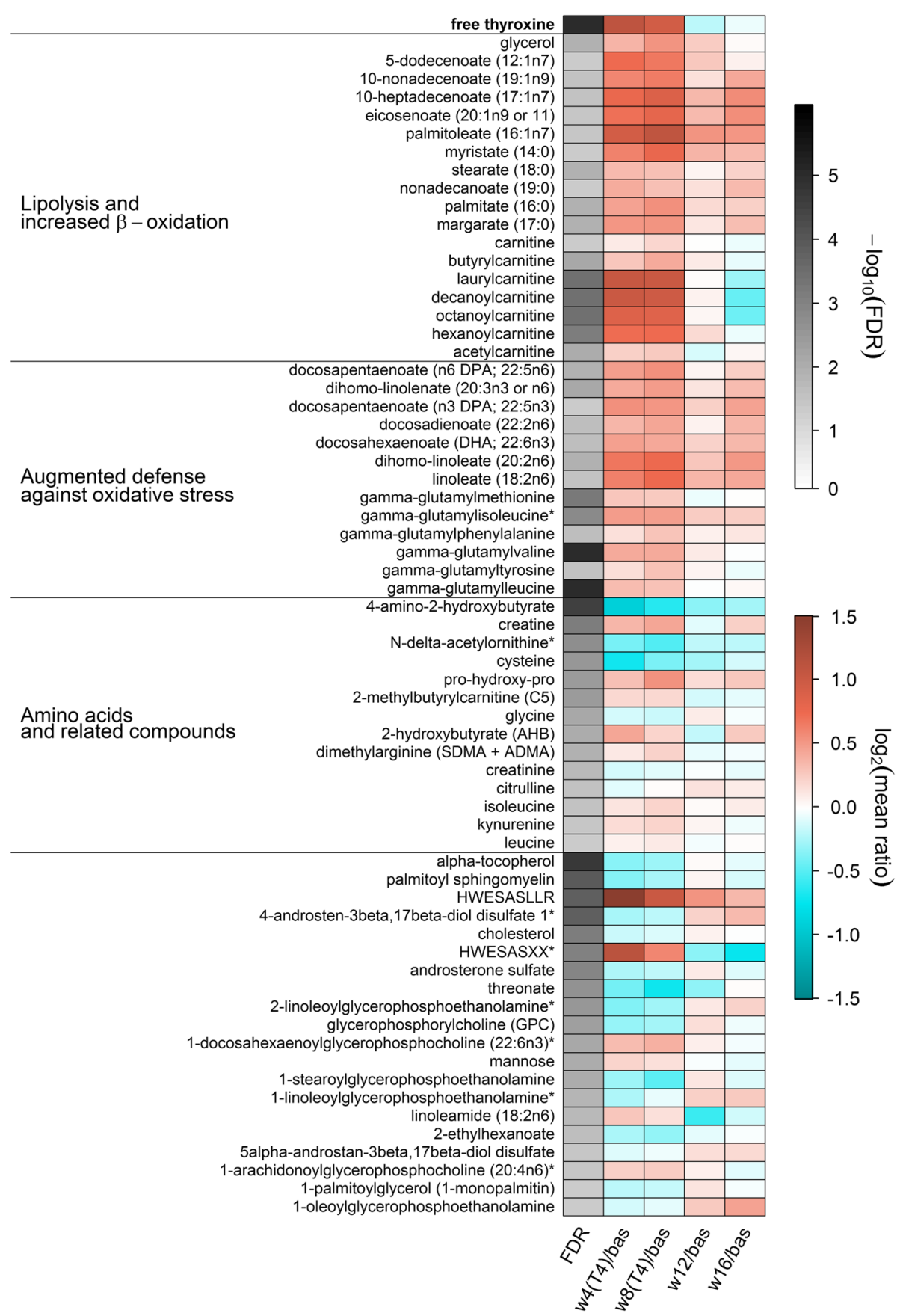

Fig. 3 Heatmap of plasma metabolites significantly associated with free thyroxine $\left(\mathrm{FT}_{4}\right)$ in mixed effect linear regression models. The first column displays the values of the mean false discovery rate (FDR) for the $\mathrm{FT}_{4}$ effect across 101 subsamples coded in grey color. The other columns indicate the mean $\log _{2}$-ratio from baseline (bas) compared to 4 (w4(T4)) and 8 (w8(T4)) weeks of treatment as well as 4 (w12) and 8 (w16) weeks after finishing the treatment. The time course of the $\mathrm{FT}_{4}$ concentrations is shown on top of the map as reference. Orange shading denotes an increase and blue shading a decrease compared to baseline, respectively. Derived physiological signatures are labeled on the left. The corresponding estimates and FDR values from regression analysis can be found in Additional file 2: Table S1. Metabolites marked with a star were assigned based on in silico fragmentation spectra 


\section{A plasma metabolome signature indicating increased resting energy expenditure and enhanced mitochondrial fatty acid $\beta$-oxidation}

Thyroxine treatment induced an increase in long chain saturated as well as monounsaturated FFAs, which was accompanied by elevated glycerol levels (Fig. 3). As demonstrated by Mitchell et al. [25], both Graves' thyrotoxicosis and resistance to $\mathrm{TH}$ due to THRB mutations $(\mathrm{RTH} \beta)$ result in significantly increased resting energy expenditure. Involved mechanisms include $\mathrm{TH}$-stimulated lipolysis in white adipose tissue, mediated by increased local concentrations of catecholamines with successive activation of adipocyte $\beta$-adrenergic receptors [26, 27]. Consistently, the ubiquitous increase of FFAs and glycerol in plasma following $\mathrm{L}-\mathrm{T}_{4}$ treatment observed in the present study clearly indicates $\mathrm{TH}$-triggered lipolysis in white adipose tissue.

After transport into tissues highly active in respiration, namely skeletal muscle as the major determinant of energy expenditure in humans [28], and liver, FFAs are subjected to mitochondrial $\beta$-oxidation that is also enhanced by $\mathrm{TH}$. This is mainly mediated by increased expression of CPT1 encoding carnitine palmitoyltransferase-I (CPT-1), which represents a direct transcriptional target of $\mathrm{T}_{3}$-activated TR [29]. In exchange with carnitine, acyl-carnitines are translocated through the outer mitochondrial membrane by CPT-1 [30]. Simultaneous TH-mediated up-regulation of the gene encoding the final enzyme of carnitine biosynthesis, the $\gamma$-butyrobetaine hydroxylase, ensures the increased carnitine levels required for that, as demonstrated in a rodent model [31]. The resulting enhanced activity of the complete carnitine acyl-carnitine translocase system explains the pronounced increase in short to medium chain ACs in plasma observed in this study (Fig. 3), as a fraction of the newly synthesized ACs spills from tissue in the circulation, as reported earlier by Jourdan et al. [32]. Indeed, our results replicate the positive association between $\mathrm{ACs}$ and $\mathrm{FT}_{4}$ reported by this group for the population-based KORA cohort study based on data from 1463 euthyroid individuals [32]. In contrast, a previous patient-based study revealed no relation between plasma AC profiles and the restoration of euthyroidism [33]. However, the value of the latter results might be limited by the small sample size of six hyper- and hypothyroid individuals, respectively.

Augmented $\beta$-oxidation of FFAs causes increased acetyl-CoA levels and stimulation of the TCA cycle, which would be expected to finally trigger increased ATP production by oxidative phosphorylation. Indeed, accelerated carbon flux through the TCA cycle was demonstrated in human skeletal muscle in a short-term model of experimental thyrotoxicosis (Lebon et al. [34]) as well as in patients suffering from RTH $\beta$ (Mitchell et al. [25]). However, ATP production by oxidative phosphorylation did not change under these conditions, which was explained by increased uncoupling of respiration and ATP synthesis, potentially caused by increased expression of UCP3 (SLC25A9) encoding the mitochondrial uncoupling protein 3 (Lebon et al. 2001; Mitchell et al. [25]). Less efficient ATP generation in muscle might also explain our observation of increased creatine plasma levels (Fig. 2) under conditions of thyrotoxicosis, as the synthesis of the central muscle energy storage compound phosphocreatine by ATP-dependent creatine phosphorylation can be predicted to be reduced by $\mathrm{TH}$-induced mitochondrial uncoupling.

Enhanced systemic glucose utilization represents a further well-known consequence of increased energy expenditure under conditions of hyperthyroidism [29]. In the liver, TH stimulate glycogenolysis and gluconeogenesis mainly consuming gluconeogenic amino acids and glycerol while down-regulating glycolysis, resulting in a higher hepatic glucose output [35]. In this context, the already mentioned strongly increased plasma glycerol pool under $\mathrm{L}_{-} \mathrm{T}_{4}$ treatment that is explained by stimulated lipolysis also represents a potential source for gluconeogenesis (Fig. 3). Our did not support for the use of amino acids as a source for TH-stimulated hepatic gluconeogenesis, partially in line with results of the already mentioned population-based KORA study [32]. However, the unaltered plasma levels of gluconeogenic amino acids that were measured despite the predicted increased demand after $\mathrm{L}_{-} \mathrm{T}_{4}$-treatment could be caused by the enhanced renal amino acid recovery reported for such conditions [36].

In skeletal muscle, TH induce expression of SLC2A4 encoding the glucose transporter GLUT4 as well as trafficking of GLUT4 to the plasma membrane [29]. Thus, increased glucose uptake in peripheral tissues, namely the muscle, might explain our observation that in spite of increased hepatic glucose release the corresponding serum concentrations were not significantly changed (Table 1). However, besides glucose, glycogenolysis also produces glucose-6-phosphate which subsequently can be converted to mannose [37]. Whether the increased plasma mannose levels observed in our study (Fig. 3) reflect THstimulated glycogenolysis in liver or result from glycogen breakdown in muscle remains unclear.

\section{A plasma metabolome signature indicating augmented defense against systemic oxidative stress}

The strong positive $\mathrm{FT}_{4}$-association observed for $\gamma$ glutamyl amino acid (GGAA) levels represents one of the novel findings of this study (Fig. 3). As elevated GGAA levels were recently related to several types of liver damage [38] and TH were furthermore demonstrated to represent potent hepatic mitogens triggering hepatocyte turnover [39], we first hypothesized that the observed increase during thyrotoxicosis might also result from hepatocellular lysis. However, determination of the classical laboratory 
markers for liver damage, namely ALT, AST, and GGT activities in serum, did not support this hypothesis.

GGAA synthesis by transfer of the glutathione (GSH) glutamyl moiety to free amino acids is catalyzed by $\gamma$ glutamyl transpeptidase (GGT) as part of the $\gamma$-glutamyl cycle (GGC). As the GGT catalytic center is localized at the extracytoplasmic side of the hepatocyte membrane, this represents the site of $\gamma$-glutamyl amino acid production, which is strongly determined by the availability of GSH [40]. The rate-limiting step in GSH biosynthesis is catalyzed by the heterodimeric glutamate-cysteine ligase consisting of a heavy catalytic and a light regulatory subunit. Expression of GCLC and GCLM encoding the two subunits of the enzyme is induced by transcriptional up-regulation via NRF2, the redox-sensitive key regulatory transcription factor of the major cellular defense system against oxidative stress [41]. Strikingly, hepatic activation of the NRF2 regulon by $\mathrm{TH}$-induced production of reactive oxygen species due to increased respiration was demonstrated in rodent models $[42,43]$. Similarly, a stimulatory effect of TH on the GGC resulting in improved antioxidant capacity was shown in astrocytes [44]. Thus, it seems plausible that the increase in plasma GGAA levels under conditions of thyrotoxicosis observed in this study reflects the NRF2-mediated induction of GSH synthesis as part of the systemic network antagonizing pronounced oxidative stress as a consequence of $\mathrm{FT}_{4}$-stimulated respiration.

Our study further revealed an $\mathrm{FT}_{4}$-associated increase in $\mathrm{n} 3$ and n6 plasma PUFAs and a drop in LPs (Fig. 3), partially replicating findings from other recent metabolome analyses [17, 32, 45]. Indeed, the LPs displayed opposite associations, depending on the presence of either choline (lysophosphatidylcholine (LPC); positively associated) or ethanolamine (lysophosphatidylethanolamine (LPE); negatively associated) as the head group (Fig. 3).

Previous animal studies demonstrated a direct negative effect of TH on the hepatic PUFA content [46-48], wherein PUFAs were depleted under hyperthyroid conditions. It was supposed that $\mathrm{TH}$ initiate remodeling of mitochondrial membranes resulting in a decrease of PUFA-containing phosphatidylcholines. As saturated FAs are less prone to peroxidation, this was interpreted as an adaptive mechanism contributing to the protection of mitochondrial membranes against enhanced oxidative stress forced by TH-induced up-regulation of respiration [46, 47]. Thus, PUFA release from mitochondrial membranes might be reflected in the observed increased plasma PUFA levels.

The negative association between serum $\mathrm{FT}_{4}$ and plasma LPEs represents an additional novel finding of this study (Fig. 3). Previous analyses of rodent hyperthyroidism models $[49,50]$ revealed enhanced incorporation of phosphatidylethanolamine (PE) in mitochondria of liver [50] and brain [49]. Augmented PE utilization might explain the observed plasma decrease of PE metabolites, namely the LPEs.

\section{Discordant changes in classical and novel markers of kidney function under thyrotoxicosis}

As outlined above, increased plasma creatine levels under conditions of thyrotoxicosis most likely reflect decreased creatine phosphorylation in skeletal muscle due to pronounced uncoupling of respiration and ATP synthesis. By contrast, we observed decreased plasma levels of the creatine catabolite creatinine in this study (Fig. 3), which could be explained by increased renal clearance of creatinine, possibly above the general glomerular filtration rate [51]. Both findings were previously described for hyperthyroidism $[52,53]$. Thus, a reliable estimation of the glomerular filtration rate based on creatinine might be biased and hampers the interpretation of kidney function in thyroid disease. Similar holds true for CYTC, the second common circulating marker for kidney function, which was strongly elevated by $\mathrm{L}-\mathrm{T}_{4}$ treatment in our study, a finding in line with previous results of hyperthyroidism studies [54, 55]. Very recently, novel promising markers for kidney function estimation were published using a similar metabolomics approach as in this study [56], namely C-mannosyltryptophan and pseudouridine. In contrast to creatinine and CYTC, none of these markers was altered to a similar extent in the present study (Additional file 2: Table S1), suggesting that the observed changes in creatinine and CYTC levels are metabolically driven and to a lesser extent due to altered kidney function. Thus, novel markers of kidney function such as C-mannosyltryptophan and pseudouridine may be advantageous under conditions of thyrotoxicosis.

\section{Thyrotoxicosis increases plasma asymmetric dimethylarginine (ADMA) levels}

We observed increased plasma levels of methylated arginine (as the sum of ADMA and symmetric dimethylarginine) under $\mathrm{L}-\mathrm{T}_{4}$ treatment, while the levels of its catabolite citrulline decreased (Fig. 3). A positive association between TH levels and circulating ADMA was previously reported in epidemiological studies and under conditions of hyperthyroidism [57-60]. ADMA was suggested as a severe risk factor for cardiovascular disease (for review see [61]), mainly as it directly inhibits endothelial nitric oxide synthase (NOS), thereby impairing NO-dependent vasodilation and favoring hypertension. ADMA is generated as a putative by-product of pronounced systemic proteolysis [62], which is known to be triggered by $\mathrm{TH}$ excess [63]. Therefore, the increased plasma levels of leucine, isoleucine, and their degradation intermediate 2-methylbutyrylcarnitine in the present study (Fig. 3) might indicate pronounced $\mathrm{FT}_{4}$-associated protein catabolism. The products of dimethylarginine dimethylaminohydrolase-catalyzed ADMA degradation, citrulline, and dimethylamine, are subsequently cleared by the kidneys [64]. At least one study using a murine model [65] described an inhibitory effect of prolonged 
$\mathrm{T}_{3}$ treatment on dimethylarginine dimethylaminohydrolase in the liver. Thus, our novel observation of an $\mathrm{FT}_{4}$-associated ADMA/symmetric dimethylarginine increase and citrulline decrease in plasma may indicate TH-induced suppression of ADMA catabolism leading to its systemic accumulation. In sum, augmented production as well as reduced decomposition of ADMA might therefore contribute to its increased plasma levels under conditions of thyrotoxicosis.

Thyrotoxicosis-induced elevated ADMA levels are predicted to mediate increased blood pressure by NOS inhibition [57-59]. The notion that TH directly affect vascular smooth muscle cells causing vascular relaxation and dilatation $[66,67]$ seemingly contradict the above discussed findings. However, it appears that the described TR $\alpha$-dependent, non-genomic activation of endothelial NOS via the PI3/AKT-pathway is only present at very high $\mathrm{TH}$ concentrations $[68,69]$, which were not observed in the present study.

General $\mathrm{FT}_{4}$-associated alterations of the plasma proteome Using an untargeted shotgun-LC-MS/MS-approach, our proteome study demonstrated two major general categories of proteins exhibiting $\mathrm{FT}_{4}$-associated plasma levels, namely the higher abundant actively secreted proteins predominantly originating from the liver and representing the majority of detected proteins and, in addition, leakage proteins whose presence in the circulation is predicted to result from cell lysis. The latter comprised only $3 \%$ of the total protein intensity. Similar to the metabolome, the levels of 63 out of 437 detected proteins (14\%) exhibited significant associations with $\mathrm{FT}_{4}$ (Additional file 2: Table S2). The majority $(\mathrm{N}=47)$ was positively associated, whereas about one fourth $(\mathrm{N}=16)$ demonstrated a negative association with serum $\mathrm{FT}_{4}$. SHBG and CYTC, which are known to be altered in thyroid dysfunction, were among the strong positively $\mathrm{FT}_{4}$-associated proteins. The changes in the levels of both proteins as determined by MS were similar to those measured with standard laboratory assays (Additional file 1: Figure S3). Of note, we observed no significant alterations of the major $\mathrm{TH}$ transport proteins thyroxine-binding globulin (SERPINA7) and thyroid-hormone binding protein transthyretin. The results are summarized in Fig. 4 and Additional file 2: Table S2.

\section{A plasma proteome signature indicating decreased lipoprotein particle levels during thyrotoxicosis}

TH-dependent alterations in the levels of apolipoproteins and different lipid-rich particles were reported previously [70-73]. In line with these findings, we observed a significant drop in the plasma levels of apolipoproteins $\mathrm{APOB}$ (apoB-100), APOD, and APOC3 (apoCIII) during the peak of induced thyrotoxicosis, where APOD exhibited the strongest association (Fig. 4 and Additional file 2: Table S2).
The apoB100 protein represents the primary apolipoprotein of VLDL and LDL particles essentially mediating systemic transport of lipids including cholesterol to peripheral tissues in the context of the fuel and overflow transport pathways, respectively, and is the primary ligand of the low-density lipoprotein receptor (LDL-R) [74]. Peripheral as well as liver-specific LDL particle uptake via apoB100-dependent LDL-R binding and endocytosis is promoted by $\mathrm{TH}$, as $L D L R$ encoding this receptor represents a direct TR target and is additionally upregulated by the transcriptional regulator SREBP-2, which, in turn, is also induced by $\mathrm{TH}$ at the gene expression level [75-77]. Thus, the decreased apoB100 abundance under conditions of thyrotoxicosis represents a direct consequence of $\mathrm{TH}$-stimulated LDL uptake from the circulation.

APOD is primarily associated with HDL particles mediating reverse cholesterol transport (RCT) from peripheral tissues to the liver. It represents an atypical apolipoprotein and belongs to the family of lipocalin proteins which transport small hydrophobic ligands [78]. TH stimulate the RCT by increasing the expression of several genes involved in cholesterol metabolism, among them SCARB1 encoding the multiple-ligand binding scavenger receptor class B member 1 (SRB1), which is responsible for the binding of cholesterol enriched HDL particles in numerous cell tissues, namely liver and adrenal [79]. Therefore, the observed drop in APOD plasma levels can be explained by $\mathrm{TH}$-stimulated HDL particle binding as part of the activated RCT.

The apoCIII protein is localized on the surface of mature triglyceride-rich chylomicrons and VLDL particles as well as HDL particles contributing to the fuel transport and the RCT pathways, respectively [74]. Uptake of VLDL particles is mediated by the VLDL receptor that binds APOE, a further apolipoprotein found in chylomicrons. Expression of the gene encoding this receptor was demonstrated to be under positive TH control in a rodent model [80]. Therefore, the decreased apoCIII levels observed in our study during thyrotoxicosis are explained by TH-mediated upregulation of the genes encoding VLDL receptor and SRB1.

The TH-induced drop in the plasma levels of apolipoproteins belonging to different classes is consistent with the observed significant $\mathrm{TH}$-associated transient reductions in the plasma levels of HDL-cholesterol, LDLcholesterol, and total cholesterol as determined by standard clinical assays (Table 1 and Fig. 2).

\section{A plasma proteome signature indicating augmented coagulation during thyrotoxicosis}

The positive association between blood coagulation and $\mathrm{TH}$ concentrations is well known. In line with the predominantly clinical studies published so far on this topic [81-85], our proteome analysis demonstrated several 


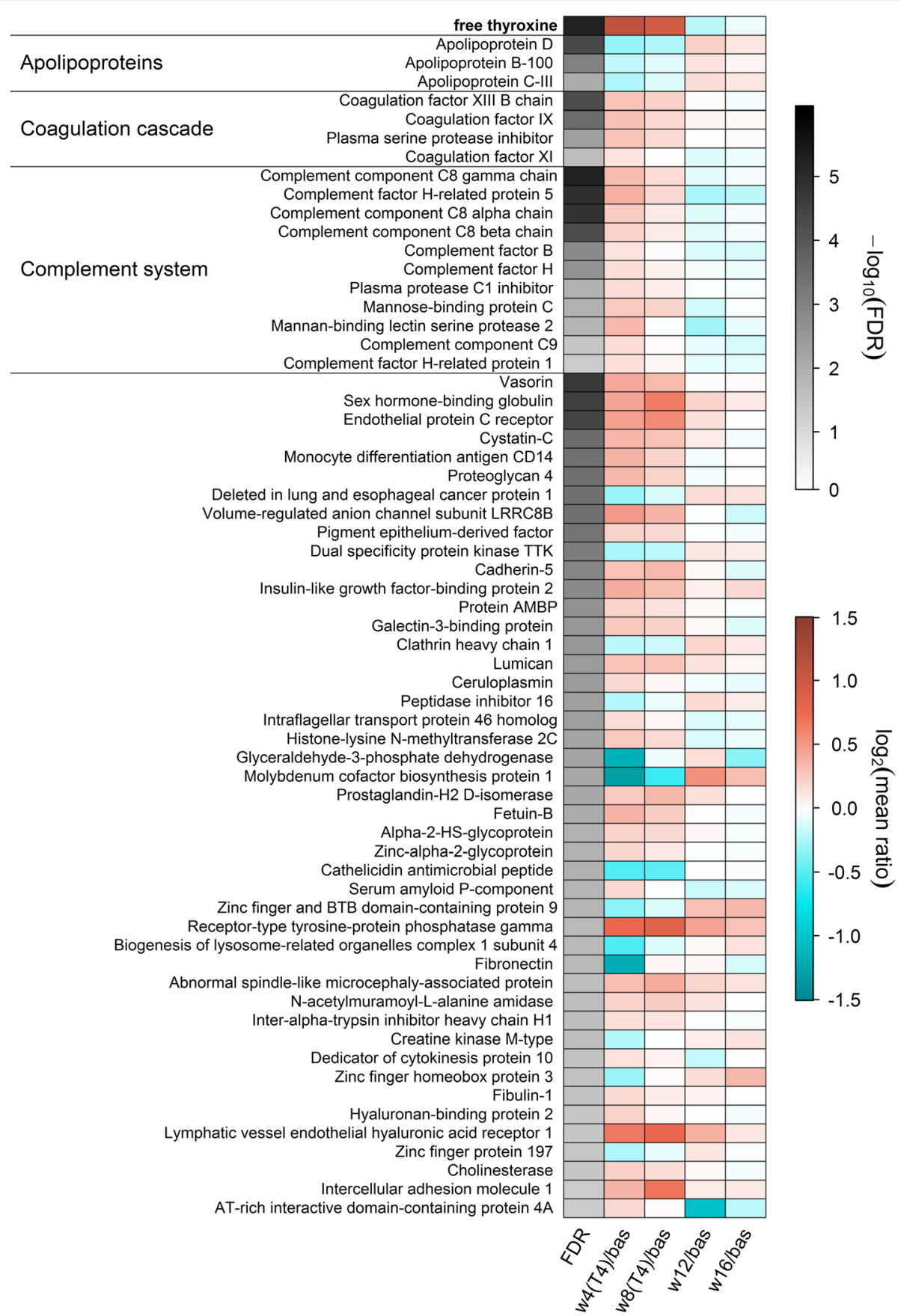

Fig. 4 Heatmap of plasma proteins significantly associated with free thyroxine $\left(\mathrm{FT}_{4}\right)$ in mixed effect linear regression models. The first column displays the values of the mean false discovery rate (FDR) for the $\mathrm{FT}_{4}$ effect across 101 subsamples of the data coded in grey color. The other columns indicate the mean $\log _{2}$-ratio from baseline (bas) compared to 4 (w4(T4)) and 8 (w8(T4)) weeks of treatment as well as 4 (w12) and 8 (w16) weeks after finishing the treatment. The time course of $\mathrm{FT}_{4}$ concentrations is shown on the top of the map as reference. Orange shading denotes an increase and blue shading a decrease compared to baseline, respectively. Derived physiological signatures are labeled on the left. The corresponding estimates and FDR values from regression analysis can be found in Additional file 2: Table S2

proteins involved in the coagulation cascade to exhibit $\mathrm{FT}_{4}$-associated plasma levels (Fig. 4); these findings have been published separately [86]. In short, we were able to demonstrate that experimental thyrotoxicosis increases the levels of coagulation cascade proteins in plasma, supporting a positive impact of $\mathrm{TH}$ on blood coagulation even at non-pathological levels. Coagulation factor XIII B chain (F13B) and the factors IX (FA9) and XI (FA11) 
exhibited significantly increased levels under thyrotoxicosis conditions, as well as SERPINA5 (IPSP), an inhibitor of activated protein $\mathrm{C}$. As the latter inhibits clot formation, increased levels of its inhibitor SERPINA5 and of factors XIIIB, IX, and XI can be predicted to result in a prothrombotic, hypercoaguable environment, in accordance with the aforementioned findings from clinical studies [81-85]. For factors XIIIB and IX, the in-

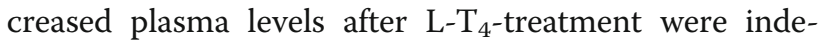
pendently validated by ELISA techniques [86]. The molecular mechanism(s) underlying the increased levels of these proteins are currently not clear and cannot be clarified by our study design. Plausible hypotheses might be a TH-stimulated increase of their stability in plasma or enhanced expression of their encoding genes. The recently published finding of unaltered coagulation parameters in RTH $\beta$ patients despite elevated $\mathrm{FT}_{4}$ levels favors the latter hypothesis and suggests that the procoagulant effects observed under conditions of thyrotoxicosis and hyperthyroidism are mediated via $\operatorname{TR} \beta$ [87].

\section{A plasma proteome signature indicating increased complement system plasma protein levels}

Nine proteins of the complement system were positively associated with $\mathrm{FT}_{4}$, including mannose-binding protein $\mathrm{C}$ (MBL2) and mannan-binding lectin serine protease 2 (MASP2). Additionally, the complement-factor $\mathrm{H}$-related proteins CFHR1 and CFHR5 as binding proteins of complement component C3b showed a positive association with serum $\mathrm{FT}_{4}$ (Fig. 4). Consistently, although the C3 protein barely missed statistical significance in the proteome analysis (Additional file 2: Table S2), higher abundance of C3 as well as C4 was determined by standard laboratory assays (Table 1 and Fig. 2). In addition, our metabolome analysis detected one C3 fragment (HWESASLLR) [88] mirroring the positive association (Fig. 3). Similarly, clinical studies $[84,89,90]$ reported positive associations between complement system proteins and $\mathrm{TH}$ levels. Of note, the duration of hyperthyroidism might be crucial in this context, since a short-term model of experimental thyrotoxicosis revealed no significant C3 level alterations [84]. Data explaining the impact of $\mathrm{TH}$ on the complement system proteins, which originate primarily from the liver, are scarce. Previous studies demonstrated that $M B L 2$ encoding mannose-binding lectin, an early module of the lectin pathway in the complement system that exhibited increased plasma levels under thyrotoxicosis conditions in our study, represents a direct PPAR $\alpha$ target $[91,92]$. As PPAR $\alpha$ belongs to those transcriptional co-regulators interacting with TH-ligated TR [29], it may be speculated that a corresponding TH-mediated induction of $M B L 2$ expression causes the observed plasma level increase, which might also hold true for other complement system proteins. However, in mice, the fact that several analyses of the hepatic transcriptome in different murine models before and after $\mathrm{T}_{3}$ application failed so far to demonstrate convincingly differentially expressed genes encoding complement system proteins [65, 93-95] argues against a TR-dependent regulation at the mRNA level. Future analyses involving TH-treated primary human hepatocytes might represent a promising approach to clarify the molecular mechanism(s) underlying the observed plasma level alterations of complement system proteins.

\section{Differences in response profiles and effect magnitudes}

Different kinetic patterns as well as mean magnitude sizes were observed for several associated molecule categories (Figs. 3 and 4): ACs exhibited an invariable response during the complete study period whereas the levels of long-chain FAs further increased between 4 and 8 weeks (Fig. 3). In contrast, complement system proteins reached their maximal mean levels after 4 weeks of $\mathrm{L}^{-} \mathrm{T}_{4}$ treatment followed by a moderate decline after 8 weeks (Fig. 4). Timedependent alterations were also evident in the magnitudes of the detected effects. For instance, lipid species demonstrated an almost two-fold increase, whereas the levels of complement system proteins increased only moderately $(\sim 30 \%)$. Despite these differences, all associations were most likely mediated by $\mathrm{L}_{-} \mathrm{T}_{4}$ treatment as they were captured by the mixed-effect linear regression models.

\section{A biomarker-based signature to predict thyroid function}

Our approach of a precisely controlled transient increase in systemic $\mathrm{TH}$ levels in healthy male volunteers allowed characterizing effects clearly and unambiguously based on a defined duration and dose of thyroxine. As we deliberately searched for a clinically relevant and yet unmet diagnostic approach to classify TH status without using TSH and/or free $\mathrm{TH}$, we combined the data generated by the untargeted OMICS techniques in a classification model. For this purpose, we built a random forest classifier via a two-stage cross-validation procedure (see Methods and Additional file 1: Figure S1), thereby allowing for a more realistic estimation of the generalization of the random forest than conventional k-fold cross-validation. This is exemplarily demonstrated by the ROC curves (Fig. 5), which were obtained when both situations occurred, perfect $($ AUC $=1$ ) as well as fair (AUC $=0.75)$ classification neither representing the expected general performance. Additionally, the approach chosen here allowed for combining classification with feature selection to define a small set of biomarkers among the numerous $\mathrm{FT}_{4}$-associated molecules described above. We obtained a list comprising 15 metabolites and proteins (Fig. 5, left panel) exhibiting robust and good classification performance over all 30 validation runs (mean $\mathrm{AUC}=0.86$; Fig. 5, right panel) and therefore representing 

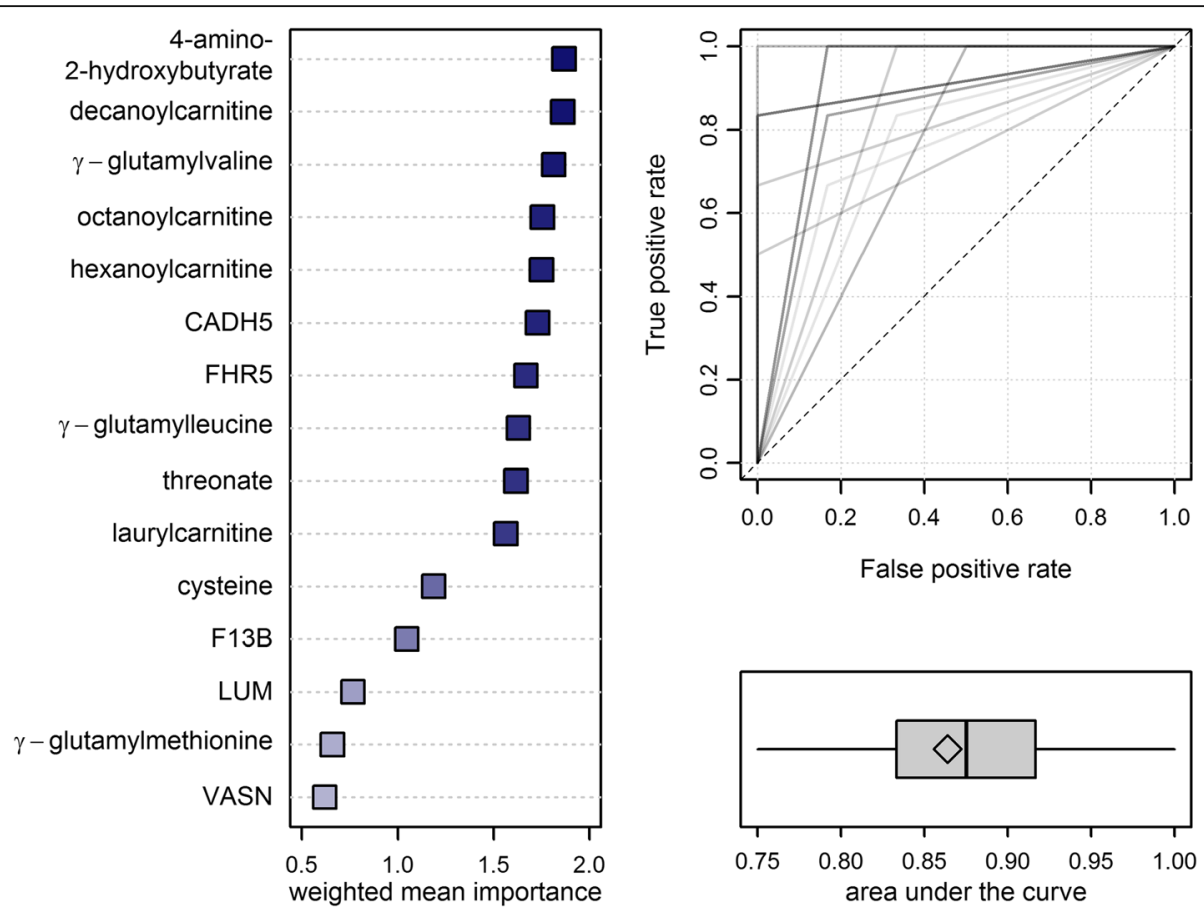

Fig. 5 Final results from classification analyses using random forests in a two-stage cross-validation scheme with 50 inner and 30 outer loops (Additional file 1: Figure S1). Left panel: Fifteen most important metabolites/proteins ranked by a weighted (area under the curve) mean Gini index. Right panel: Receiver operating characteristic (ROC) curves (upper) and boxplot of the area under the curve (lower) from 30 outer loops. Overlapping ROCs are displayed by darker shades and the diamond indicates the mean AUC. CADH5 cadherin-5, FHR5 complement factor H-related protein 5, F13B coagulation factor XIII B chain, LUM lumican, VASN vasorin

appropriate biomarkers for a reliable prediction of the $\mathrm{TH}$ status. Of this biomarker panel, most components were already discussed above in the context of different physiological signatures, namely increased resting energy expenditure and enhanced mitochondrial fatty acid $\beta$-oxidation (decanoylcarnitine, octanoylcarnitine, hexanoylcarnitine, laurylcarnitine), increased GSH synthesis as part of the augmented defense against oxidative stress as a consequence of stimulated respiration $(\gamma$-glutamylvaline, $\gamma$-glutamylleucine, $\gamma$-glutamylmethionine), and increased levels of complement system (FHR5) or coagulation (F13B) proteins.

Cadherin-5 (CDH5), also named VE-cadherin, represents a calcium-dependent adhesion protein specific to endothelial cells and is a major component of adherens junctions found in blood vessels. It is essentially involved in the regulation of vascular integrity, restricting permeability of the endothelium [96]. Shedding of CDH5 by proteinases increases microvascular permeability and is one of the molecular mechanisms involved in transendothelial neutrophil migration during inflammatory processes and endothelial apoptosis [97]. CDH5 shedding is mediated by two proteinases of the ADAM (A Disintegrin And a Metalloprotease domain metalloproteases) family, namely ADAM9 and ADAM10 [98], where the proteolytic activity of ADAM10 is stimulated by thrombin (coagulation factor 2, F2a) [99]. Therefore, the observed increased levels of circulating
CDH5 most probably result from stimulated thrombin activation under the prothrombotic thyrotoxicosis-associated conditions described above.

A similar explanation can be found for the increased vasorin (VASN) levels - VASN is a typical type I membrane protein that was identified as target of the ADAM17 metalloprotease. Limited proteolysis by ADAM17 generates a soluble fragment encompassing the extracellular VASN domain, which directly binds to transforming growth factor (TGF) $\beta$ and attenuates TGF $\beta$ signaling [100]. As in the case of ADAM10, ADAM17-dependent shedding has been demonstrated to be stimulated by thrombin [101]. Consistently, the three CDH5-specific peptides as well as the six VASN-specific peptides that were detected in the proteome analysis exclusively mapped to the extracellular domains of the two proteins. Therefore, together with CFHR5, CDH5, and VASN can be assigned to the signature of increased levels of coagulation factors.

The plasma level of cysteine was previously reported to be decreased by $\mathrm{TH}$-treatment via direct down-regulation of the expression of $C T H$ and $C B S$ encoding cystathionine gamma-lyase and cystathionine beta-synthase, respectively [102]. This might explain the pronounced negative association between the plasma levels of cysteine as well as its metabolite 4-amino-2-hydroxybutyrate and $\mathrm{FT}_{4}$ in our 
study (Fig. 3). Threonate, which is either derived from glycated proteins or represents a degradation product of ascorbate [103], was previously linked to altered thyroid function in two murine models $[45,104]$, but not yet in humans.

Finally, lumican (LUM) represents an extracellular matrix protein modified as a proteoglycan in several tissues. The core protein with leucine-rich repeats that are characteristic for the corresponding superfamily binds collagen fibrils and regulates its structure. In addition, LUM associates with CD14 on the surface of macrophages and neutrophils and promotes the CD14-TLR4 mediated response to bacterial lipopolysaccharides where it is involved in macrophagemediated phagocytosis [105-107]. LUM is proteolytically degraded by matrix metalloproteinase (MMP) 14 [108], whose encoding gene $M M P 14$, among several other genes encoding MMPs, was demonstrated to be down-regulated by TH [109]. Thus, the observed increase in plasma LUM levels might be the consequence of its decreased degradation due to $\mathrm{TH}$-mediated reduced MMP14 expression.

\section{Conclusion}

The unique feature of the present study is its specific experimental design. By focusing on healthy young male volunteers we excluded any potentially interfering, disease-specific influences related, for example, to the autoimmune process in Graves' thyrotoxicosis. This approach allows to control the extent and duration of biochemical hyperthyroidism induced by thyroxine as one of the most prescribed drugs and to monitor its recovery, thereby enabling unique insights into the kinetics of subjective changes experienced by the volunteers in relation to the biochemical changes measured.

Application of $\mathrm{L}-\mathrm{T}_{4}$ clearly induced biochemical thyrotoxicosis in our volunteer sample as indicated by strongly suppressed TSH and clearly increased TH levels, which were rapidly reversed after ceasing hormone application. The model was further validated by known thyrotoxic alterations in biochemical markers, including SHBG, CYTC, and total and LDL-cholesterol [12, 13, 55].

Interestingly, as described in a previous study analyzing the same volunteer sample, subjectively the volunteers did not notice any thyrotoxic symptoms, which was supported by the negative results of a standardized questionnaire for thyrotoxicosis and a battery of behavioral and cognitive tests [24]. A 24-h blood pressure profile before and at the end of 8 weeks of thyroxine application was not significantly different, neither when analyzed over $24 \mathrm{~h}$ or during sleep between 01:00 and 06:00 am [24]. In contrast, comparison of the mean 24-h pulse rates revealed a moderate increase of about 8.9 beats/minute (from 66.7 to 75.6 beats/minute), which was not recognized by the volunteers [24].

$\mathrm{L}-\mathrm{T}_{4}$ application clearly changed a large number of biochemical parameters beyond the expected alterations in
TSH and TH levels or in known biochemical markers. The combined findings reveal a surprising discrepancy between biochemical alterations and subjective symptoms in this cohort of young healthy subjects and shed new light on the mechanisms mediating adaptation to subclinical hyperthyroidism. They suggest that biochemical alterations might be detectable considerably earlier than clinical symptoms occur and are much more sensitive. In consequence, the diagnosis of thyrotoxicosis in clinical routine might frequently miss the real onset time of the disease by several months.

The comprehensive robust analysis of a human thyrotoxicosis model using state of the art untargeted plasma OMICS approaches demonstrated a strong and pleiotropic metabolic impact of $\mathrm{TH}$ on this compartment. The characterized physiological signatures comprised biomarkers indicating increased resting energy expenditure, augmented defense against systemic oxidative stress, decreased lipoprotein particle levels, and increased levels of complement system proteins as well as coagulation factors, where the latter results in a pro-thrombotic environment. Measurement of 15 specific biomarkers, metabolites as well as proteins, allowed reliable prediction of the individual thyroid function in the analyzed sample independent of common TSH and $\mathrm{FT}_{4}$ measurements. In addition, by following the subjects during the recovery from thyrotoxicosis we gained first insight in the target-specific kinetics of TH-dependent responses. The definition of this prediction panel might represent an important step forward in molecular characterization of early forms of hyperthyroidism. However, it has to be emphasized that the analyzed study sample consisted exclusively of young men, limiting the generalizability of the results. Therefore, further validation studies using larger samples of higher complexity in terms of age and sex as well as hypothyroid conditions have to be performed. Similarly, our findings concerning the influence of increased $\mathrm{TH}$ levels on kidney function markers and systemic ADMA levels that might be of special clinical importance have to be replicated in appropriate patient cohorts and/or population-based studies where the required parameters are available.

\section{Additional files}

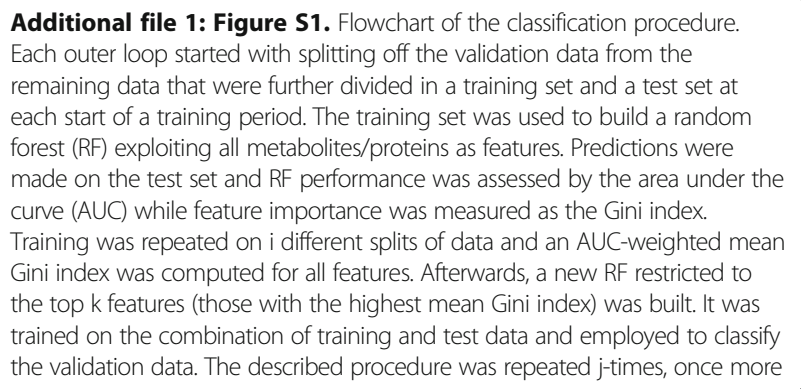

Additional file 1: Figure S1. Flowchart of the classification procedure. Each outer loop started with splitting off the validation data from the remaining data that were further divided in a training set and a test set at each start of a training period. The training set was used to build a random forest (RF) exploiting all metabolites/proteins as features. Predictions were made on the test set and RF performance was assessed by the area under the curve (AUC) while feature importance was measured as the Gini index. Training was repeated on i different splits of data and an AUC-weighted mean Gini index was computed for all features. Afterwards, a new RF restricted to the top $k$ features (those with the highest mean Gini index) was built. It was trained on the combination of training and test data and employed to classify the validation data. The described procedure was repeated j-times, once more 
yielding an AUC-weighted mean Gini index for feature importance. The final results of this procedure with $i=50, j=30$ and $k=15$ are shown in Fig. 4 in the main text. Figure S2. Boxplots for each study time point for glucose and total cholesterol levels measured either by standard laboratory assays (dark grey) or by metabolomics (light grey). bas baseline, w4(T4)/W8(T4) 4 and 8 weeks of levothyroxine treatment; w12/w16 4 and 8 weeks after stopping the application. Figure $\mathbf{S 3}$. Boxplots for each study time point for sexhormone- binding globulin (SHBG) and cystatin C levels determined either by standard laboratory assays (dark grey) or in the untargeted proteome approach (light grey). bas baseline, w4(T4)/W8(T4) 4 and 8 weeks of levothyroxine treatment, w12/W16 4 and 8 weeks after stopping the application. (DOCX 580 $\mathrm{kb})$

Additional file 2: Table S1. Results from mixed-effect linear regression analyses with serum FT4 concentrations as exposure and metabolites as outcome. Table S2. Results from mixed-effect linear regression analyses with serum FT4 concentrations as exposure and protein levels as outcome. (XLSX 88 kb)

\section{Abbreviations}

AC: acyl carnitines; ADMA: asymetric dimethylarginine; ALT: alanine amino transferase; APOB: apolipoprotein B100; APOC3: apolipoprotein C3; APOD: apolipoprotein D; AST: aspartate amnio transferase; AUC: area under the curve; CDH5: cadherin-5; CPT-I: carnitine palmitoyltransferase-l; CYTC: cystatin C; FDR: false discovery rate; FFA: free fatty acids; $\mathrm{FT}_{3}$ : (free) triiodothyronine; $\mathrm{FT}_{4}$ : (free) thyroxine; GC: gas chromatography; GGAA: $\gamma^{-}$ glutamyl amino acid; GGC: $y$-glutamyl cycle; GGT: $\gamma$-glutamyl transpeptidase; GSH: glutathione; HDL: high-density lipoprotein; LC: liquid chromatography; LDL: low-density lipoprotein; LP: Iysolipids; LPC: Iysophosphatidylcholine; LPE: lysophosphatidylethanolamine; L-T4: levothyroxine; LUM: Iumican; MS: mass spectrometry; NOS: nitric oxide synthase;

PE: phosphatidylethanolamine; PUFA: poly unsaturated fatty acids; RCT: reverse cholesterol transport; ROC: receiver operating characteristics; RTHB: resistance to thyroid hormone; SHBG: sex hormone-binding globulin; TH: thyroid hormones; TR: thyroid hormone receptor; TRH: thyrotropin releasing hormone; TSH: thyrotropin; VASN: vasorin; VLDL: very low-density lipoprotein

\section{Abbreviations}

AC: acyl carnitines; ADMA: asymetric dimethylarginine; ALT: alanine amino transferase; APOB: apolipoprotein B100; APOC3: apolipoprotein C3; APOD: apolipoprotein D; AST: aspartate amnio transferase; AUC: area under the curve; CDH5: cadherin-5; CPT-l: carnitine palmitoyltransferase-l; CYTC: cystatin C; FDR: false discovery rate; FFA: free fatty acids; $\mathrm{FT}_{3}$ : (free) triiodothyronine; $\mathrm{FT}_{4}$ : (free) thyroxine; GC: gas chromatography; GGAA: $\gamma$-glutamyl amino acid; GGC: $\gamma$-glutamyl cycle; GGT: $\gamma$-glutamyl transpeptidase; GSH: glutathione; HDL: high-density lipoprotein; LC: liquid chromatography; LDL: low-density lipoprotein; LP: Iysolipids; LPC: lysophosphatidylcholine;

LPE: Iysophosphatidylethanolamine; L-T: levothyroxine; LUM: lumican; MS: mass spectrometry; NOS: nitric oxide synthase; PE: phosphatidylethanolamine; PUFA: poly unsaturated fatty acids; RCT: reverse cholesterol transport; ROC: receiver operating characteristics; RTH $\beta$ : resistance to thyroid hormone; SHBG: sex hormone-binding globulin; TH: thyroid hormones; TR: thyroid hormone receptor; TRH: thyrotropin releasing hormone; TSH: thyrotropin; VASN: vasorin; VLDL: very low-density lipoprotein;

\section{Acknowledgements}

We thank all study participants whose personal dedication and commitment made this project possible.

\section{Funding}

Analyses were supported by grants from the German Research Foundation as part of the priority program "Thyroid Trans Act" (DFG WA 1328/5-1, MU 3811/16-2, FR 3055/4-1, VO 1444/9-1, BR 915/14-1) and by grant FU 356/7-1 to DF. NF received funding from the European Union's Horizon 2020 research and innovation program under the Marie Skłodowska-Curie Grant Agreement number 657595.

\section{Availability of data and materials}

The mass spectrometry proteomics data have been deposited to the ProteomeXchange Consortium via the PRIDE [20] partner repository with the dataset identifier PXD004815 and 10.6019/PXD004815. All other datasets analyzed during the current study are available from the corresponding authors on reasonable request.

\section{Authors' contributions}

MP contributed to data evaluation, analyses and interpretation of data, and wrote the manuscript. BE performed proteome measurements and wrote the manuscript. TK designed the classification scheme and contributed to computational analyses. JG contributed to data interpretation. AD, KAI and TM contributed to the study design and sample collection. EH supervised proteome measurements and contributed to the interpretation of the data. $\mathrm{GH}$ contributed to interpretation of the data and writing of the manuscript. $\mathrm{GB}, \mathrm{HW}, \mathrm{UV}$, and NF initiated the study and contributed to data evaluation and writing of the manuscript. MN and NF provided laboratory measurements data. NF further contributed to computational analyses. DF critical reviewed the manuscript and assisted in data interpretation. All authors read and approved the final manuscript.

\section{Competing interests}

The authors declare that they have no competing interests.

\section{Consent for publication}

Not applicable.

\section{Ethics approval and consent to participate}

The work has been approved by the ethics committee of the University of Lübeck and written informed consent was received from all participants prior to the study. The study conformed to the WMA Declaration of Helsinki.

\section{Author details}

${ }^{1}$ Institute of Clinical Chemistry and Laboratory Medicine, University Medicine Greifswald, Ferdinand-Sauerbruch-Straße, 17475 Greifswald, Germany. ${ }^{2}$ DZHK (German Centre for Cardiovascular Research), partner site Greifswald, Greifswald, Germany. ${ }^{3}$ Department of Functional Genomics, Interfaculty Institute for Genetics and Functional Genomics, University Medicine and Ernst-Moritz-Arndt University Greifswald, Friedrich-Ludwig-Jahn-Straße 15a, D-17475 Greifswald, Germany. ${ }^{4}$ Medical Clinic I, University of Lübeck, Experimental and Clinical Endocrinology, Ratzeburger Allee 160, Zentralklinikum (Haus 40), 23538 Lübeck, Germany. ${ }^{5}$ Private Practice Endocrinology, Krämpferstraße 6, 99094 Erfurt, Germany. ${ }^{6}$ Department of Endocrinology and Metabolism, University Hospital Essen, University Duisburg-Essen, Hufelandstraße 55, 45122 Essen, Germany. ${ }^{7}$ Department of Neurology, University of Lübeck, Ratzeburger Allee 169, 23538 Lübeck, Germany. ${ }^{8}$ Research Centre for Prevention and Health, Glostrup University Hospital, Nordre Ringvej 57, 2600 Glostrup, Denmark. ${ }^{9}$ ZIK-FunGene (Zentrum für Innovationskompetenz - Funktionelle Genomforschung), Greifswald, Germany.

Received: 10 September 2016 Accepted: 15 December 2016 Published online: 09 January 2017

\section{References}

1. Yen PM. Physiological and molecular basis of thyroid hormone action. Physiol Rev. 2001;81(3):1097-142.

2. Visser TJ. Thyroid hormone transporters and resistance. Endocr Dev. 2013;24: 1-10. doi:10.1159/000343695

3. Davis PJ, Goglia F, Leonard JL. Nongenomic actions of thyroid hormone. Nat Rev Endocrinol. 2016;12(2):111-21. doi:10.1038/nrendo.2015.205.

4. Pearce SH, Brabant G, Duntas LH, Monzani F, Peeters RP, Razvi S, et al. 2013 ETA guideline: management of subclinical hypothyroidism. Eur Thyroid J. 2013;2(4):215-28. doi:10.1159/000356507.

5. Andersen S, Pedersen KM, Bruun NH, Laurberg P. Narrow individual variations in serum $T(4)$ and $T(3)$ in normal subjects: a clue to the understanding of subclinical thyroid disease. J Clin Endocrinol Metab. 2002;87(3):1068-72. doi:10.1210/jcem.87.3.8165.

6. Benhadi N, Fliers E, Visser TJ, Reitsma JB, Wiersinga WM. Pilot study on the assessment of the setpoint of the hypothalamus-pituitary-thyroid axis in healthy volunteers. Eur J Endocrinol. 2010;162(2):323-9. doi:10.1530/eje-09-0655.

7. Medici M, Visser WE, Visser TJ, Peeters RP. Genetic determination of the hypothalamic-pituitary-thyroid axis: where do we stand? Endocr Rev. 2015;36(2):214-44. doi:10.1210/er.2014-1081. 
8. Thienpont LM, Van Uytfanghe K, Beastall G, Faix JD, leiri T, Miller WG, et al. Report of the IFCC Working Group for Standardization of Thyroid Function Tests; part 1: thyroid-stimulating hormone. Clin Chem. 2010;56(6):902-11.

9. Thienpont LM, Van Uytfanghe K, Beastall G, Faix JD, leiri T, Miller WG, et al. Report of the IFCC Working Group for Standardization of Thyroid Function Tests; part 2: free thyroxine and free triiodothyronine. Clin Chem. 2010;56(6):912-20.

10. Ortiga-Carvalho TM, Sidhaye AR, Wondisford FE. Thyroid hormone receptors and resistance to thyroid hormone disorders. Nat Rev Endocrinol. 2014; 10(10):582-91. doi:10.1038/nrendo.2014.143.

11. Gurnell M, Halsall DJ, Chatterjee VK. What should be done when thyroid function tests do not make sense? Clin Endocrinol (Oxf). 2011;74(6):673-8. doi:10.1111/j.1365-2265.2011.04023.x.

12. Thaler MA, Seifert-Klauss V, Luppa PB. The biomarker sex hormone-binding globulin - from established applications to emerging trends in clinical medicine. Best Pract Res Clin Endocrinol Metab. 2015;29(5):749-60. doi:10.1016/j.beem.2015.06.005.

13. Razvi S, Ingoe L, Keeka G, Oates C, McMillan C, Weaver JU. The beneficial effect of L-thyroxine on cardiovascular risk factors, endothelial function, and quality of life in subclinical hypothyroidism: randomized, crossover trial. J Clin Endocrinol Metab. 2007;92(5):1715-23. doi:10.1210/jc.2006-1869.

14. Jonklaas J, Bianco AC, Bauer AJ, Burman KD, Cappola AR, Celi FS, et al. Guidelines for the treatment of hypothyroidism: prepared by the american thyroid association task force on thyroid hormone replacement. Thyroid. 2014;24(12):1670-751. doi:10.1089/thy.2014.0028.

15. Silvestri E, Coppola M, Cioffi F, Goglia F. Proteomic approaches for the study of tissue specific effects of 3,5,3'-triiodo-L-thyronine and 3,5-diiodo-Lthyronine in conditions of altered energy metabolism. Front Physiol. 2014;5:491. doi:10.3389/fphys.2014.00491.

16. Sinha RA, You SH, Zhou J, Siddique MM, Bay BH, Zhu X, et al. Thyroid hormone stimulates hepatic lipid catabolism via activation of autophagy. J Clin Invest. 2012;122(7):2428-38. doi:10.1172/jci60580.

17. Wu S, Gao Y, Dong X, Tan G, Li W, Lou Z, et al. Serum metabonomics coupled with ingenuity pathway analysis characterizes metabolic perturbations in response to hypothyroidism induced by propylthiouracil in rats. J Pharm Biomed Anal. 2013;72:109-14. doi:10.1016/j.jpba.2012.09.030.

18. Wu S, Tan G, Dong X, Zhu Z, Li W, Lou Z, et al. Metabolic profiling provides a system understanding of hypothyroidism in rats and its application. PLoS One. 2013;8(2):e55599.

19. Evans AM, DeHaven CD, Barrett T, Mitchell M, Milgram E. Integrated, nontargeted ultrahigh performance liquid chromatography/electrospray ionization tandem mass spectrometry platform for the identification and relative quantification of the small-molecule complement of biological systems. Anal Chem. 2009;81(16):6656-67. doi:10.1021/ac901536h.

20. Vizcaino JA, Csordas A, del-Toro N, Dianes JA, Griss J, Lavidas I, et al. 2016 update of the PRIDE database and its related tools. Nucleic Acids Res. 2016:44(D1):D447-56. doi:10.1093/nar/gkv1145.

21. Benjamini $Y$, Hochberg $Y$. Controlling the false discovery rate: a practical and powerful approach to multiple testing. J R Stat Soc Ser B (Methodological). 1995;57(1):289-300.

22. Liaw A, Wiener M. Classification and regression by randomForest. R News. 2002;2(3):18-22

23. Breiman L, Friedman JH, Olshen RA, Stone CJ. Classification and regression trees. New York: Chapman Hall; 1984.

24. Gottlich M, Heldmann M, Gobel A, Dirk AL, Brabant G, Munte TF. Experimentally induced thyrotoxicosis leads to increased connectivity in temporal lobe structures: a resting state $\mathrm{fMRI}$ study. Psychoneuroendocrinology. 2015;56:100-9. doi:10.1016/.jpsyneuen.2015.03.009.

25. Mitchell CS, Savage DB, Dufour S, Schoenmakers N, Murgatroyd P, Befroy D, et al. Resistance to thyroid hormone is associated with raised energy expenditure, muscle mitochondrial uncoupling, and hyperphagia. J Clin Invest. 2010;120(4):1345-54. doi:10.1172/jci38793.

26. Nedvidkova J, Haluzik M, Bartak V, Dostalova I, Vlcek P, Racek P, et al. Changes of noradrenergic activity and lipolysis in the subcutaneous abdominal adipose tissue of hypo- and hyperthyroid patients: an in vivo microdialysis study. Ann N Y Acad Sci. 2004;1018:541-9. doi:10.1196/annals.1296.067.

27. Viguerie $\mathrm{N}$, Langin D. Effect of thyroid hormone on gene expression. Curr Opin Clin Nutr Metab Care. 2003;6(4):377-81. doi:10.1097/01.mco. 0000078998.96795.e7.

28. Zurlo F, Larson K, Bogardus C, Ravussin E. Skeletal muscle metabolism is a major determinant of resting energy expenditure. J Clin Invest. 1990;86(5): 1423-7. doi:10.1172/jci114857.
29. Mullur R, Liu YY, Brent GA. Thyroid hormone regulation of metabolism. Physiol Rev. 2014;94(2):355-82. doi:10.1152/physrev.00030.2013.

30. Ramsay RR. The carnitine acyltransferases: modulators of acyl-CoAdependent reactions. Biochem Soc Trans. 2000;28(2):182-6.

31. Galland S, Georges B, Le Borgne F, Conductier G, Dias JV, Demarquoy J. Thyroid hormone controls carnitine status through modifications of gamma-butyrobetaine hydroxylase activity and gene expression. Cell Mol Life Sci. 2002;59(3):540-5.

32. Jourdan C, Linseisen J, Meisinger C, Petersen A-K, Gieger C, Rawal R, et al. Associations between thyroid hormones and serum metabolite profiles in an euthyroid population. Metabolomics. 2014;10(1):152-64. doi:10.1007/ s11306-013-0563-4.

33. Wong S, Hannah-Shmouni F, Sinclair G, Sirrs S, Dahl M, Mattman A. Acylcarnitine profile in thyroid disease. Clin Biochem. 2013;46(1-2):180-3. doi:10.1016/j.clinbiochem.2012.10.006.

34. Lebon V, Dufour S, Petersen KF, Ren J, Jucker BM, Slezak LA, et al. Effect of triiodothyronine on mitochondrial energy coupling in human skeletal muscle. J Clin Invest. 2001;108(5):733-7. doi:10.1172/jci11775.

35. Mitrou P, Raptis SA, Dimitriadis G. Insulin action in hyperthyroidism: a focus on muscle and adipose tissue. Endocr Rev. 2010;31(5):663-79. doi:10.1210/ er.2009-0046.

36. Pietzner M, Budde K, Homuth G, Völker U, Völzke H, Nauck M, et al. Distinct urinary metabolic profiles associated with serum TSH and FT4 concentrations. Metabolomics. 2015;11(5):1316-26. doi:10.1007/s11306-015-0788-5.

37. Taguchi T, Yamashita E, Mizutani T, Nakajima H, Yabuuchi M, Asano N, et al. Hepatic glycogen breakdown is implicated in the maintenance of plasma mannose concentration. Am J Physiol Endocrinol Metab. 2005;288(3):E534-40. doi:10.1152/ajpendo.00451.2004.

38. Soga T, Sugimoto M, Honma M, Mori M, Igarashi K, Kashikura K, et al. Serum metabolomics reveals gamma-glutamyl dipeptides as biomarkers for discrimination among different forms of liver disease. J Hepatol. 2011;55(4): 896-905. doi:10.1016/j.jhep.2011.01.031.

39. Pibiri M, Ledda-Columbano GM, Cossu C, Simbula G, Menegazzi M, Shinozuka $\mathrm{H}$, et al. Cyclin D1 is an early target in hepatocyte proliferation induced by thyroid hormone (T3). FASEB J. 2001;15(6):1006-13.

40. Griffith OW, Meister A. Glutathione: interorgan translocation, turnover, and metabolism. Proc Natl Acad Sci U S A. 1979;76(11):5606-10.

41. Wild AC, Moinova HR, Mulcahy RT. Regulation of gamma-glutamylcysteine synthetase subunit gene expression by the transcription factor Nrf2. J Biol Chem. 1999;274(47):33627-36.

42. Cornejo P, Vargas R, Videla LA. Nrf2-regulated phase-ll detoxification enzymes and phase-III transporters are induced by thyroid hormone in rat liver. BioFactors. 2013;39(5):514-21. doi:10.1002/biof.1094

43. Romanque $P$, Cornejo P, Valdes S, Videla LA. Thyroid hormone administration induces rat liver Nrf2 activation: suppression by $\mathrm{N}$ acetylcysteine pretreatment. Thyroid. 2011;21(6):655-62. doi:10.1089/thy. 2010.0322.

44. Dasgupta A, Das S, Sarkar PK. Thyroid hormone promotes glutathione synthesis in astrocytes by up regulation of glutamate cysteine ligase through differential stimulation of its catalytic and modulator subunit mRNAs. Free Radic Biol Med. 2007;42(5):617-26. doi:10.1016/j.freeradbiomed.2006.11.030.

45. Montoya GA, Strauss V, Fabian E, Kamp H, Mellert W, Walk T, et al. Mechanistic analysis of metabolomics patterns in rat plasma during administration of direct thyroid hormone synthesis inhibitors or compounds increasing thyroid hormone clearance. Toxicol Lett. 2014;225(2):240-51. doi:10.1016/j.toxlet.2013.12.010.

46. Guerrero A, Pamplona R, Portero-Otin M, Barja G, Lopez-Torres M. Effect of thyroid status on lipid composition and peroxidation in the mouse liver. Free Radic Biol Med. 1999;26(1-2):73-80.

47. Hoch FL. Lipids and thyroid hormones. Prog Lipid Res. 1988;27(3):199-270.

48. Raederstorff D, Meier CA, Moser U, Walter P. Hypothyroidism and thyroxin substitution affect the n-3 fatty acid composition of rat liver mitochondria. Lipids. 1991;26(10):781-7.

49. Bangur CS, Howland JL, Katyare SS. Thyroid hormone treatment alters phospholipid composition and membrane fluidity of rat brain mitochondria. Biochem J. 1995;305(Pt 1):29-32.

50. Ruggiero FM, Landriscina C, Gnoni GV, Quagliariello E. Lipid composition of liver mitochondria and microsomes in hyperthyroid rats. Lipids. 1984;19(3):171-8.

51. Shirota T, Shinoda T, Yamada T, Aizawa T. Alteration of renal function in hyperthyroidism: increased tubular secretion of creatinine and decreased distal tubule delivery of chloride. Metabolism. 1992;41(4):402-5. 
52. Carter WJ, van der Weijden Benjamin WS, Faas FH. Mechanism of creatinuria in experimental hyperthyroidism. Acta Endocrinol (Copenh). 1981;97(4):479-85.

53. Verhelst J, Berwaerts J, Marescau B, Abs R, Neels H, Mahler C, et al. Serum creatine, creatinine, and other guanidino compounds in patients with thyroid dysfunction. Metabolism. 1997;46(9):1063-7.

54. Jayagopal V, Keevil BG, Atkin SL, Jennings PE, Kilpatrick ES. Paradoxical changes in cystatin $C$ and serum creatinine in patients with hypo- and hyperthyroidism. Clin Chem. 2003;49(4):680-1.

55. Wiesli P, Schwegler B, Spinas GA, Schmid C. Serum cystatin C is sensitive to small changes in thyroid function. Clin Chim Acta. 2003;338(1-2):87-90.

56. Sekula P, Goek ON, Quaye L, Barrios C, Levey AS, Romisch-Margl W, et al. A metabolome-wide association study of kidney function and disease in the general population. J Am Soc Nephrol. 2016;27(4):1175-88. doi:10.1681/asn. 2014111099.

57. Arikan E, Karadag CH, Guldiken S. Asymmetric dimethylarginine levels in thyroid diseases. J Endocrinol Investig. 2007;30(3):186-91. doi:10.1007/ bf03347423.

58. Gu LQ, Zhao L, Zhu W, Li FY, Zhang MJ, Liu Y, et al. Relationships between serum levels of thyroid hormones and serum concentrations of asymmetric dimethylarginine (ADMA) and N-terminal-pro-B-type natriuretic peptide (NTproBNP) in patients with Graves' disease. Endocrine. 2011;39(3):266-71. doi:10.1007/s12020-011-9436-7.

59. Hermenegildo C, Medina P, Peiro M, Segarra G, Vila JM, Ortega J, et al. Plasma concentration of asymmetric dimethylarginine, an endogenous inhibitor of nitric oxide synthase, is elevated in hyperthyroid patients. J Clin Endocrinol Metab. 2002;87(12):5636-40. doi:10.1210/jc.2002-020905.

60. Ittermann T, Bahls M, Atzler D, Friedrich N, Schwedhelm E, Boger RH, et al. L-Arginine derivatives are associated with the hyperthyroid state in the general population. Thyroid. 2016;26(2):212-8. doi:10.1089/thy.2015.0385

61. Willeit P, Freitag DF, Laukkanen JA, Chowdhury S, Gobin R, Mayr M, et al. Asymmetric dimethylarginine and cardiovascular risk: systematic review and meta-analysis of 22 prospective studies. J Am Heart Assoc. 2015;4(6): e001833. doi:10.1161/jaha.115.001833.

62. Boger $\mathrm{RH}$. The emerging role of asymmetric dimethylarginine as a novel cardiovascular risk factor. Cardiovasc Res. 2003;59(4):824-33.

63. Muller MJ, Seitz HJ. Thyroid hormone action on intermediary metabolism. Part III. Protein metabolism in hyper- and hypothyroidism. Klin Wochenschr. 1984;62(3):97-102.

64. Hu X, Atzler D, Xu X, Zhang P, Guo H, Lu Z, et al. Dimethylarginine dimethylaminohydrolase-1 is the critical enzyme for degrading the cardiovascular risk factor asymmetrical dimethylarginine. Arterioscler Thromb Vasc Biol. 2011;31(7):1540-6. doi:10.1161/atvbaha.110.222638.

65. Flores-Morales A, Gullberg H, Fernandez L, Stahlberg N, Lee NH, Vennstrom $B$, et al. Patterns of liver gene expression governed by TRbeta. Mol Endocrinol. 2002;16(6):1257-68. doi:10.1210/mend.16.6.0846.

66. Ojamaa K, Klemperer JD, Klein I. Acute effects of thyroid hormone on vascular smooth muscle. Thyroid. 1996;6(5):505-12.

67. Bussemaker E, Popp R, Fisslthaler B, Larson CM, Fleming I, Busse R, et al. Hyperthyroidism enhances endothelium-dependent relaxation in the rat renal artery. Cardiovasc Res. 2003;59(1):181-8.

68. Hiroi $Y$, Kim HH, Ying H, Furuya $F$, Huang $Z$, Simoncini $T$, et al. Rapid nongenomic actions of thyroid hormone. Proc Natl Acad Sci U S A. 2006;103(38):14104-9. doi:10.1073/pnas.0601600103.

69. Lozano-Cuenca J, Lopez-Canales OA, Aguilar-Carrasco JC, Villagrana-Zesati JR, Lopez-Mayorga RM, Castillo-Henkel EF, et al. Pharmacological study of the mechanisms involved in the vasodilator effect produced by the acute application of triiodothyronine to rat aortic rings. Brazil J Med Biol Res. 2016;49(8): doi:10.1590/1414-431×20165304.

70. de Bruin TW, van Barlingen $\mathrm{H}$, van Linde-Sibenius TM, van Vuurst de Vries AR, Akveld MJ, Erkelens DW. Lipoprotein(a) and apolipoprotein B plasma concentrations in hypothyroid, euthyroid, and hyperthyroid subjects. J Clin Endocrinol Metab. 1993;76(1):121-6. doi:10.1210/jcem.76.1.8421075.

71. Lee WY, Suh JY, Rhee EJ, Park JS, Sung KC, Kim SW. Plasma CRP, apolipoprotein A-1, apolipoprotein B and Lpa levels according to thyroid function status. Arch Med Res. 2004;35(6):540-5. doi:10.1016/j.arcmed.2004.08.003.

72. Muls E, Blaton V, Rosseneu M, Lesaffre E, Lamberigts $G$, De Moor P. Serum lipids and apolipoproteins $A-I, A-I I$, and B in hyperthyroidism before and after treatment. J Clin Endocrinol Metab. 1982;55(3):459-64. doi:10.1210/jcem-55-3-459.

73. O'Brien T, Katz K, Hodge D, Nguyen TT, Kottke BA, Hay ID. The effect of the treatment of hypothyroidism and hyperthyroidism on plasma lipids and apolipoproteins Al, All and E. Clin Endocrinol. 1997;46(1):17-20.
74. Dominiczak MH, Caslake MJ. Apolipoproteins: metabolic role and clinical biochemistry applications. Ann Clin Biochem. 2011;48(Pt 6):498-515. doi:10.1258/acb.2011.011111.

75. Coppola M, Glinni D, Moreno M, Cioffi F, Silvestri E, Goglia F. Thyroid hormone analogues and derivatives: Actions in fatty liver. World J Hepatol. 2014;6(3):114-29. doi:10.4254/wjh.v6.i3.114.

76. Lopez D, Abisambra Socarras JF, Bedi M, Ness GC. Activation of the hepatic LDL receptor promoter by thyroid hormone. Biochim Biophys Acta. 2007; 1771(9):1216-25. doi:10.1016/j.bbalip.2007.05.001.

77. Shin DJ, Osborne TF. Thyroid hormone regulation and cholesterol metabolism are connected through Sterol Regulatory Element-Binding Protein-2 (SREBP-2). J Biol Chem. 2003;278(36):34114-8. doi:10.1074/jbc.M305417200.

78. Rassart E, Bedirian A, Do Carmo S, Guinard O, Sirois J, Terrisse L, et al. Apolipoprotein D. Biochim Biophys Acta. 2000;1482(1-2):185-98.

79. Sinha RA, Singh BK, Yen PM. Thyroid hormone regulation of hepatic lipid and carbohydrate metabolism. Trends Endocrinol Metab. 2014;25(10):538-45. doi:10.1016/j.tem.2014.07.001.

80. Jokinen EV, Landschulz KT, Wyne KL, Ho YK, Frykman PK, Hobbs HH. Regulation of the very low density lipoprotein receptor by thyroid hormone in rat skeletal muscle. J Biol Chem. 1994;269(42):26411-8.

81. Van Zaane B, Squizzato A, Debeij J, Dekkers OM, Meijers JC, Van Zanten AP, et al. Alterations in coagulation and fibrinolysis after levothyroxine exposure in healthy volunteers: a controlled randomized crossover study. J Thromb Haemost. 2011;9(9):1816-24. doi:10.1111/j.1538-7836.2011.04430.x.

82. Stuijver DJ, van Zaane B, Romualdi E, Brandjes DP, Gerdes VE, Squizzato A. The effect of hyperthyroidism on procoagulant, anticoagulant and fibrinolytic factors: a systematic review and meta-analysis. Thromb Haemost. 2012;108(6):1077-88. doi:10.1160/th12-07-0496.

83. Squizzato A, Romualdi E, Buller HR, Gerdes VE. Clinical review: thyroid dysfunction and effects on coagulation and fibrinolysis: a systematic review. J Clin Endocrinol Metab. 2007;92(7):2415-20. doi:10.1210/jc.2007-0199.

84. Hooper JM, Stuijver DJ, Orme SM, van Zaane B, Hess K, Gerdes VE, et al. Thyroid dysfunction and fibrin network structure: a mechanism for increased thrombotic risk in hyperthyroid individuals. J Clin Endocrinol Metab. 2012;97(5):1463-73. doi:10.1210/jc.2011-2894.

85. Debeij J, van Zaane B, Dekkers OM, Doggen CJ, Smit JW, van Zanten AP, et al. High levels of procoagulant factors mediate the association between free thyroxine and the risk of venous thrombosis: the MEGA study. J Thromb Haemost. 2014;12(6):839-46. doi:10.1111/jth.12573.

86. Engelmann B, Bischof J, Dirk AL, Friedrich N, Hammer E, Thiele T, et al. Effect of experimental thyrotoxicosis onto blood coagulation: a proteomics study. Eur Thyroid J. 2015;4 Suppl 1:119-24. doi:10.1159/000381769.

87. Elbers LP, Moran C, Gerdes VE, van Zaane B, Meijers J, Endert E, et al. The hypercoagulable state in hyperthyroidism is mediated via the thyroid hormone beta receptor pathway. Eur J Endocrinol. 2016. Ahead of print. doi:10.1530/eje-15-1249.

88. Dousset B, Straczek J, Maachi F, Nguyen DL, Jacob C, Capiaumont J, et al. Purification from human plasma of a hexapeptide that potentiates the sulfation and mitogenic activities of insulin-like growth factors. Biochem Biophys Res Commun. 1998;247(3):587-91. doi:10.1006/bbrc.1998.8834.

89. Zhang B, Jiang Y, Yang Y, Peng F, Hu X. Correlation between serum thyroxine and complements in patients with multiple sclerosis and neuromyelitis optica. Neuro Endocrinol Lett. 2008;29(2):256-60.

90. Potlukova E, Jiskra J, Freiberger T, Limanova Z, Zivorova D, Malickova K, et al The production of mannan-binding lectin is dependent upon thyroid hormones regardless of the genotype: a cohort study of 95 patients with autoimmune thyroid disorders. Clin Immunol. 2010;136(1):123-9. doi:10.1016/j.clim.2010.02.015.

91. Rakhshandehroo M, Stienstra R, de Wit NJ, Bragt MC, Haluzik M, Mensink RP, et al. Plasma mannose-binding lectin is stimulated by PPARalpha in humans. Am J Physiol Endocrinol Metab. 2012;302(5):E595-602. doi:10.1152/ajpendo.00299.2011.

92. Tachibana K, Takeuchi K, Inada H, Sugimoto K, Ishimoto K, Yamashita M, et al. Human mannose-binding lectin 2 is directly regulated by peroxisome proliferator-activated receptors via a peroxisome proliferator responsive element. J Biochem. 2013;154(3):265-73. doi:10.1093/jb/mvt050.

93. Feng $X$, Jiang $Y$, Meltzer $P$, Yen PM. Thyroid hormone regulation of hepatic genes in vivo detected by complementary DNA microarray. Mol Endocrinol. 2000;14(7):947-55. doi:10.1210/mend.14.7.0470.

94. Sadow PM, Chassande O, Koo EK, Gauthier K, Samarut J, Xu J, et al. Regulation of expression of thyroid hormone receptor isoforms and coactivators in liver and heart by thyroid hormone. Mol Cell Endocrinol. 2003;203(1-2):65-75 
95. Weitzel JM, Radtke C, Seitz HJ. Two thyroid hormone-mediated gene expression patterns in vivo identified by CDNA expression arrays in rat. Nucleic Acids Res. 2001;29(24):5148-55.

96. Giannotta M, Trani M, Dejana E. VE-cadherin and endothelial adherens junctions: active guardians of vascular integrity. Dev Cell. 2013;26(5):441-54. doi:10.1016/j.devcel.2013.08.020.

97. Wallez Y, Huber P. Endothelial adherens and tight junctions in vascular homeostasis, inflammation and angiogenesis. Biochim Biophys Acta. 2008;1778(3):794-809. doi:10.1016/j.bbamem.2007.09.003.

98. Dreymueller D, Pruessmeyer J, Groth E, Ludwig A. The role of ADAMmediated shedding in vascular biology. Eur J Cell Biol. 2012;91(6-7):472-85. doi:10.1016/j.ejcb.2011.09.003.

99. Schulz B, Pruessmeyer J, Maretzky T, Ludwig A, Blobel CP, Saftig P, et al ADAM10 regulates endothelial permeability and T-cell transmigration by proteolysis of vascular endothelial cadherin. Circ Res. 2008;102(10):1192-201. doi:10.1161/circresaha.107.169805.

100. Malapeira J, Esselens C, Bech-Serra JJ, Canals F, Arribas J. ADAM17 (TACE) regulates TGFbeta signaling through the cleavage of vasorin. Oncogene. 2011;30(16):1912-22. doi:10.1038/onc.2010.565.

101. Le Gall SM, Maretzky T, Issuree PD, Niu XD, Reiss K, Saftig P, et al. ADAM17 is regulated by a rapid and reversible mechanism that controls access to its catalytic site. J Cell Sci. 2010;123(Pt 22):3913-22. doi:10.1242/jcs.069997.

102. Miguel RN, Sanders J, Furmaniak J, Smith BR, Li C, Menconi F, et al. 15th International Thyroid Congress Program and Meeting Abstracts. Thyroid. 2015;25(S1):P-1-A-337.

103. Harding JJ, Hassett PC, Rixon KC, Bron AJ, Harvey DJ. Sugars including erythronic and threonic acids in human aqueous humour. Curr Eye Res. 1999;19(2):131-6.

104. Constantinou C, Chrysanthopoulos PK, Margarity M, Klapa MI. GC-MS metabolomic analysis reveals significant alterations in cerebellar metabolic physiology in a mouse model of adult onset hypothyroidism. J Proteome Res. 2011;10(2):869-79. doi:10.1021/pr100699m.

105. Shao H, Lee S, Gae-Scott S, Nakata C, Chen S, Hamad AR, et al. Extracellular matrix lumican promotes bacterial phagocytosis, and Lum-/- mice show increased Pseudomonas aeruginosa lung infection severity. J Biol Chem. 2012;287(43):35860-72. doi:10.1074/jbc.M112.380550.

106. Shao H, Scott SG, Nakata C, Hamad AR, Chakravarti S. Extracellular matrix protein lumican promotes clearance and resolution of Pseudomonas aeruginosa keratitis in a mouse model. PLoS One. 2013;8(1):e54765. doi:10.1371/journal.pone.0054765.

107. Wu F, Vij N, Roberts L, Lopez-Briones S, Joyce S, Chakravarti S. A novel role of the lumican core protein in bacterial lipopolysaccharide-induced innate immune response. J Biol Chem. 2007;282(36):26409-17. doi:10.1074/jbc.M702402200.

108. Li Y, Aoki T, Mori Y, Ahmad M, Miyamori H, Takino T, et al. Cleavage of lumican by membrane-type matrix metalloproteinase-1 abrogates this proteoglycan-mediated suppression of tumor cell colony formation in soft agar. Cancer Res. 2004;64(19):7058-64. doi:10.1158/0008-5472.can-04-1038.

109. Tomic-Canic M, Stojadinovic O, Lee B, Walsh R, Blumenberg M. Nexus between epidermolysis bullosa and transcriptional regulation by thyroid hormone in epidermal keratinocytes. Clin Transl Sci. 2008;1(1):45-9. doi:10.1111/j.1752-8062.2008.00015.x.

\section{Submit your next manuscript to BioMed Central and we will help you at every step:}

- We accept pre-submission inquiries

- Our selector tool helps you to find the most relevant journal

- We provide round the clock customer support

- Convenient online submission

- Thorough peer review

- Inclusion in PubMed and all major indexing services

- Maximum visibility for your research

Submit your manuscript at www.biomedcentral.com/submit
Biomed Central 